\title{
Decompositions of Regular Representations of the Canonical Commutation Relations
}

By

\author{
Reinhard SCHAFLITZEL*
}

\begin{abstract}
Every cyclic regular representation of the canonical commutation relations over any inner product space $V$ can be decomposed into a direct integral of irreducible regular representations, where the fibers are representations over subspaces of $V$. An example using the so-called directproduct representations shows that generally the irreducible representations cannot be defined over the whole $V$. So we get a new type of decomposition having no equivalent in the decompositions of locally compact groups.
\end{abstract}

\section{Introduction}

In this paper we are concerned with the decomposition of regular representations of the canonical commutation relations over a complex inner product space into irreducible representations. This article is the abridged version of a paper ([10]) accepted as a doctoral thesis by the Technische Universität München (1988).

Let $V$ be a complex inner product space. A mapping $W$ of $V$ into the group $\mathscr{U}(H)$ of the unitary operators on the complex Hilbert space $H$ is called a representation of the canonical commutation relations (CCR) over $V$, iff

$$
W(f) W(g)=\exp (i \operatorname{Im}\langle f, g\rangle / 2) W(f+g) \quad \text { for } f, g \in V .
$$

$W$ is called regular, iff

$$
t \in \mathbb{R} \longmapsto W(t f) \in \mathscr{U}(H)
$$

is strongly continuous for every $f \in V$. In some cases, more general spaces $V$ are permitted (see 2.8(ii)).

If $V$ is finite dimensional, the decomposition theory for representations of

\footnotetext{
* Communicated by H. Araki, April 3, 1990.

Institut für Mathematik der Technischen Universität München, Arcisstraße 21, D-8000 München

2, Federal Republic of Germany.
} 
the $\mathbb{C C R}$ over $V$ is very simple: $\mathbb{B y}$ a famous theorem due to Stone and von Neumann, there is only one irreducible regular representation of the $\mathbb{C C R}$ over $V$, and every regular representation over $V$ is a multiple of this representation.

In G.C. Hegerfeldt's papers [4] and [5], certain regular representations of the $\mathbb{C C R}$ over infinite dimensional $V$ are decomposed into a direct integral of irreducible regular representations; for example, representations over an inner product space with countable Hamel base are allowed, but representations over the separable Hilbert space are not considered. In this paper we decompose cyclic regular representations $W: V \rightarrow \mathscr{U}(H)$ over any complex inner product space $V$ into irreducible representations:

$W$ can be decomposed into a direct integral $\int_{X}^{\oplus} W(\varphi) d v(\varphi)$, where for $\varphi \in X$ $W(\varphi)$ is an irreducible regular representation of the $\mathrm{CCR}$ over a complex subspace $V(\varphi)$ of $V$. (What this means precisely, see Chapter 1.) As to the proof we shall have to decompose a representation of a $C^{*}$-algebra called $\mathscr{B}(V)$. Since $\mathscr{B}(V)$ is nonseparable, we will not use the usual reduction theory. Instead, for the sake of applying a decomposition theorem due to R.W. Henrichs, we need the concept of direct integrals of Hilbert spaces introduced by $\mathbb{W}$. Wils (see [12]). The main difference to the usual concept is that nonseparable Hilbert spaces are permitted.

In Chapter 1 we derive the theorem above, in Chapter 2 we discuss our result :

By the theorem the representation $W$ can be decomposed into simpler components, namely into irreducible representations over complex subspaces of $V$. So all the regular representations of the CCR over $V$ are known, if we know the irreducible representations over all the subspaces of $V$. In Chapter 2 we discuss the question whether it is possible to choose the representations $W(\varphi)$ of the fibers as irreducible regular representations over the whole $V$.

We construct some examples of irreducible regular representations of the $\mathbb{C C R}$ over a dense subspace of $l^{2}(\mathbb{N})$ which cannot be extended to regular representations of the $\mathrm{CCR}$ over $l^{2}(\mathbb{N})$. They belong to the class of direct product representations introduced by $\mathbb{J} . \mathbb{R}$. Klauder, J. McKenna, and $\mathbb{E} . J$. Woods (see [7]). By forming the direct integral of suitable representations of that kind, we get a regular representation $W_{Y}$ of the $C C R$ over $l^{2}(\mathbb{N})$ with the following property: There is no decomposition $W_{Y}=\int_{Z}^{\oplus} W(\zeta) d \mu(\zeta)$ of $W_{Y}$ into irreducible regular representations $W(\zeta)$ that are defined over $l^{2}(\mathbb{N})$ - at least, if we assume that the diagonal algebra in the decomposition is maximal abelian in $\left\{W_{Y}(f): f \in l^{2}(\mathbb{N})\right\}^{\prime}$. 
So we recognize that we cannot strengthen our theorem by choosing irreducible regular representations over $V$ in the fibers.

Throughout this paper we use the following

Notation. For a Hilbert space $H$ and a subset $M$ of $H$, let be

$$
\begin{aligned}
& H_{1}:=\{\psi \in H:\|\psi\|=1\}, \\
& M^{\perp}:=\{\psi \in H:\langle\psi, \varrho\rangle=0 \text { for every } \varrho \in M\},
\end{aligned}
$$

[M] the closed subspace of $H$, generated by $M$,

$\mathscr{L}(H)$ the set of continuous linear operators in $H$,

$\mathscr{U}(H)$ the set of unitary operators in $H$, and

具 or $\mathbb{1}_{H}$ the unit operator in $H$.

For a set $\mathscr{M} \subseteq \mathscr{L}(H)$, let be

$\mathscr{M}^{\prime} \quad$ the commutant of $\mathscr{M}$.

For a $C^{*}$-algebra $\mathscr{A}$, let be

$\mathscr{A}_{+} \quad$ the set of the positive operators of $\mathscr{A}$, and

$\mathscr{S}(\mathscr{A}) \quad$ the set of states of $\mathscr{A}$.

For $\omega \in \mathscr{S}(\mathscr{A})$, let be

$\left(\pi_{\omega}, H_{\omega}, \xi_{\omega}\right) \quad$ the corresponding GNS representation.

For a group $G$, a function $h: G \rightarrow \mathbb{C}$, and $s \in G$, let be ${ }_{s} h: G \rightarrow \mathbb{C}, \quad$ defined by $\left({ }_{s} h\right)(t):=h\left(s^{-1} \circ t\right)$.

For a set $A$ and a subset $T$ of $A$, let $\chi_{T}$ be the mapping from $A$ to $\mathbb{C}$, such that

$$
\chi_{T}(a)=\left\{\begin{array}{ll}
1 & \text { for } a \in T \\
0 & \text { for } a \notin T
\end{array} .\right.
$$

For an inner product space $V$, let be

$\mathscr{A}(V) \quad$ the CCR algebra over $V$. (This is a $C^{*}$-algebra generated by unitary operators $w(f), f \in V$, which satisfy the commutation relations.)

We note that - in an obvious manner - there is a bijective correspondence between the nondegenerate representations of $\mathscr{A}(V)$ and the representations of the CCR over $V$.

Chapter 1 . A Decomposition Theorem for Regular Representations of the Camonical Commutation Relations

\section{\$1. The $C^{*}$-Algebra $\mathscr{B}(V)$ and their Representations}

Let $V$ be an inner product space. The set $\mathbb{R} \times V$ endowed with the multiplication

$$
(s, f) \circ(t, g)=(s+t+\operatorname{Im}\langle f, g\rangle / 2, f+g)
$$


is a group called $\mathscr{H}_{V}$. By the assignment

$$
\pi \longmapsto W_{\pi}, W_{\pi}(f)=\pi(0, f),
$$

we get a bijective correspondence between the unitary representations of $\mathscr{H}_{V}$ satisfying the relations $\pi(s, 0)=e^{i s} \mathbb{1}$ for $s \in \mathbb{R}$ and the representations of the $\mathbb{C C R}$ over $V$.

If $V$ has finite dimension $m, \mathscr{H}_{V}$, carrying the usual topology, is a locally compact group, namely the Heisenberg group of dimension $m$. A representation of the CCR over $V$ is regular, iff the corresponding representation of $\mathscr{H}_{V}$ is strongly continuous.

Let $W: V \rightarrow \mathscr{U}(H)$ be a regular cyclic representation of the $\mathbb{C C R}$ over the inner product space $V$.

Let $\mathcal{N}$ be the set of the one-dimensional complex subspaces of $V$. For $N \in \mathcal{N}$ the restriction $\left.W\right|_{N}$ of $W$ to $N$ is a regular representation of the CCR over $N$. By $\pi_{N}$ we denote the corresponding representation of $\mathscr{H}_{N}$, and by $\pi_{N}^{\prime}$ the nondegenerate representation of $L^{1}\left(\mathscr{H}_{N}\right)$ associated with $\pi_{N}$ (see [2], 13.3).

Definition 1.1. The $C^{*}$-algebra in $\mathscr{L}(H)$ generated by

$$
\bigcup_{N \in \mathcal{N}} \pi_{N}^{\prime}\left(L^{1}\left(\mathscr{H}_{N}\right)\right) \cup\left\{\mathbb{1}_{H}\right\}
$$

is called $\mathscr{B}(V)$.

Remarks. (i) Let $\tilde{W}$ be another regular representation of the CCR over $V$. As before, we introduce the $C^{*}$-algebra $\tilde{\mathscr{B}}(V)$. Using the theorem due to Stone and von Neumann, we easily see that $\mathscr{B}(V)$ and $\tilde{\mathscr{B}}(V)$ are isomorphic in a canonical way.

(ii) For $N \in \mathcal{N}, L^{1}\left(\mathscr{H}_{N}\right)$ is separable. If the dimension of $V$ is greater than $1, \mathscr{N}$ is not countable, and it can be shown that $\mathscr{B}(V)$ is nonseparable. Thus, for decomposing the identity representation of $\mathscr{B}(V)$, we cannot use the classical reduction theory. If $V$ has a countable Hamel base $\left\{b_{n}: n \in \mathbb{N}\right\}$ (but not, if $V$ is a separable Hilbert space), we are able to apply the classical theory by considering the separable $C^{*}$-algebra, generated by

$$
\bigcup_{n=1}^{\infty} \pi_{\mathbb{C} b_{n}}^{\prime}\left(L^{1}\left(\mathscr{H}_{\mathbb{C} b_{n}}\right)\right) \cup\left\{\mathbb{1}_{H}\right\}
$$

instead of $\mathscr{B}(V)$, for example (cf. the proof of Theorem 2.1 in [4]).

(iii) We have

$$
\mathscr{B}(V)^{\prime}=\bigcap_{N \in \mathcal{N}}\left(\pi_{N}^{\prime}\left(L^{1}\left(\mathscr{H}_{N}\right)\right)^{\prime}=\bigcap_{N \in \mathcal{N}}\{W(f): f \in N\}^{\prime}=\{W(f): f \in V\}^{\prime} .\right.
$$


For a two-dimensional subspace $M$ of $V$ we introduce the representations $\pi_{M}$ of $\mathscr{H}_{M}$ and $\pi_{M}^{\prime}$ of $L^{1}\left(\mathscr{H}_{M}\right)$ as before. We note that for one- or twodimensional subspaces $L$ of $V$ the product of the Lebesgue measures of $\mathbb{R}$ and $L$ is the Haar measure of $\mathscr{H}_{L}$ in the normalization we will use.

Lemma 1.2. Let $N_{0}, N_{1} \in \mathscr{N}$ such that $N_{0} \neq N_{1}$, and $M:=N_{0}$ $+N_{1}$. Then the closure $\mathscr{V}\left(N_{0}, N_{1}\right)$ of the subspace in $\mathscr{L}(H)$, generated by

$$
\left\{\pi_{N_{0}}^{\prime}(h) \pi_{N_{1}}^{\prime}(k): h \in L^{1}\left(\mathscr{H}_{N_{0}}\right), k \in L^{1}\left(\mathscr{H}_{N_{1}}\right)\right\}
$$

is equal to the closure $\overline{\pi_{M}^{\prime}\left(L^{1}\left(\mathscr{H}_{M}\right)\right)}$ of $\pi_{M}^{\prime}\left(L^{1}\left(\mathscr{H}_{M}\right)\right)($ closures with respect to the norm topology).

Particularly, we have $\pi_{M}^{\prime}\left(L^{1}\left(\mathscr{H}_{M}\right)\right) \subseteq \mathscr{B}(V)$.

Proof. For one- and two-dimensional subspaces $L$ of $V$ and $r \in L^{1}(L)$, we define

$$
\bar{\pi}_{L}(r):=\int_{L} r(f) W(f) d f,
$$

By an easy consideration we get:

$$
\bar{\pi}_{L}\left(L^{1}(L)\right)=\pi_{L}^{\prime}\left(L^{1}\left(\mathscr{H}_{L}\right)\right)
$$

For $r \in L^{1}\left(N_{0}\right)$ and $s \in L^{1}\left(N_{1}\right)$ we have

$$
\begin{aligned}
\bar{\pi}_{N_{0}}(r) \bar{\pi}_{N_{1}}(s)= & \int_{N_{0}} \int_{N_{1}} r(f) s(g) W(f) W(g) d f d g \\
= & \int_{N_{0}} \int_{N_{1}}(\exp (i \operatorname{Im}\langle f, g\rangle / 2) r(f) s(g)) W(f+g) d f d g \\
& \in \bar{\pi}_{M}\left(L^{1}(M)\right)=\pi_{M}^{\prime}\left(L^{1}\left(\mathscr{H}_{M}\right)\right) .
\end{aligned}
$$

If follows $\mathscr{V}\left(N_{0}, N_{1}\right) \subseteq \overline{\pi_{M}^{\prime}\left(L^{1}\left(\mathscr{H}_{M}\right)\right)}$. Now let us prove the other inclusion.

Using a Stone-Weierstrass argument, we easily conclude that the subspace of $L^{1}\left(N_{0} \times N_{1}\right)$, generated by

$$
\left\{(f, g) \longmapsto r(f) s(g): r \in L^{1}\left(N_{0}\right), s \in L^{1}\left(N_{1}\right)\right\},
$$

is dense in $L^{1}\left(N_{0} \times N_{1}\right)$. Since the map

$$
u \longmapsto \tau(u), \tau(u)(f, g)=\exp (i \operatorname{Im}\langle f, g\rangle / 2) u(f, g),
$$

is an isometric isomorphism in $L^{1}\left(N_{0} \times N_{1}\right)$, the subspace generated by

$$
\left\{(f, g) \longmapsto \exp (i \operatorname{Im}\langle f, g\rangle / 2) r(f) s(g): r \in L^{1}\left(N_{0}\right), s \in L^{1}\left(N_{1}\right)\right\}
$$

is dense in $L^{1}\left(N_{0} \times N_{1}\right)$, too. Now it follows from relation (2) that every 
element of $\pi_{M}^{\prime}\left(L^{1}\left(\mathscr{H}_{M}\right)\right)$ can be approximated by elements of the linear span of $\left\{\bar{\pi}_{N_{0}}(r) \bar{\pi}_{N_{1}}(s): r \in L^{1}\left(N_{0}\right), s \in L^{1}\left(N_{1}\right)\right\}$.

From now on, let $\rho: \mathscr{B}(V) \rightarrow \mathscr{L}(K)$ be a nondegenerate representation of $\mathscr{B}(V), \rho_{L}^{\prime}:=\rho \circ \pi_{L}^{\prime}$ for one- or two-dimensional complex subspaces $L$ of $V$,

$\mathscr{N}_{\rho}:=\left\{N \in \mathscr{N}: \rho_{N}^{\prime}\right.$ a nondegenerate representation of $\left.L^{1}\left(\mathscr{H}_{N}\right)\right\}$, and

$$
V_{\rho}:=\left\{\begin{array}{ll}
\bigcup_{N \in \mathcal{N}_{\rho}} N & \text { for } \mathscr{N}_{\rho} \neq \emptyset \\
\{0\} & \text { for } \mathscr{N}_{\rho}=\emptyset
\end{array} \quad(\subseteq V) .\right.
$$

Proposition 1.3. (i) For $N \in \mathscr{N}_{\rho}$ let $\rho_{N}$ be the representation of $\mathscr{H}_{N}$ corresponding to $\rho_{N}^{\prime}$. Then for $t \in \mathbb{R} \rho_{N}(t, 0)=e^{i t} \mathbb{1}$, that means that a regular representation called $W_{\rho, N}$ of the CCR over $N$ is associated with $\rho_{N}$.

(ii) If $\mathcal{N}_{\rho} \neq \emptyset, V_{\rho}$ is a complex subspace of $V$; by $W_{\rho}(f):=W_{\rho, N}(f)$ for $f \in N$ and $N \in \mathcal{N}_{\rho}$, a regular representation $W_{\rho}: V_{\rho} \rightarrow \mathscr{U}(K)$ of the CCR over $V_{\rho}$ is defined.

Additionally, if $\mathscr{N}_{\rho}=\emptyset, W_{\rho}$ is defined over $V_{\rho}=\{0\}$ by $W_{\rho}(0):=\mathbb{1}_{K}$.

Proof. (i) Since for $h \in L^{1}\left(\mathscr{H}_{N}\right)$

$$
\rho\left(e^{i t} \pi_{N}^{\prime}(h)\right)=\rho\left(\pi_{N}(t, 0) \cdot \pi_{N}^{\prime}(h)\right)=\rho\left(\pi_{N}^{\prime}\left(_{(t, 0)} h\right)\right),
$$

$e^{i t} \mathbb{1}$ satisfies the equation $e^{i t} \mathbb{1} \cdot \rho_{N}^{\prime}(h)=\rho_{N}^{\prime}\left({ }_{(t, 0)} h\right)$ for $h \in L^{1}\left(\mathscr{H}_{N}\right)$. From this we get $e^{i t} \mathbb{1}=\rho_{N}(t, 0)$.

(ii) Let $N_{0}, N_{1} \in N_{\rho}$ such that $N_{0} \neq N_{1}$ and put $M:=N_{0}+N_{1}$. The representations $\rho_{N_{0}}^{\prime}$ of $L^{1}\left(\mathscr{H}_{N_{0}}\right)$ and $\rho_{N_{1}}^{\prime}$ of $L^{1}\left(\mathscr{H}_{N_{1}}\right)$ are nondegenerate. Now, considering Lemma 1.2 we see that $\rho_{M}^{\prime}$ is a nondegenerate representation of $L^{1}\left(\mathscr{H}_{M}\right)$. For every one-dimensional subspace $N$ of $M, \rho_{N}^{\prime}$ is nondegenerate. This follows for $N \neq N_{0}$ and $N \neq N_{1}$ by applying Lemma 1.2 once more (if we use $N$ instead of $N_{0}$ ). Now it is easy to show that $V_{\rho}$ is a subspace of $V$.

It is clear that $W_{\rho}$ is well defined, and that $t \in \mathbb{R} \mapsto W_{\rho}(t f)$ is strongly continuous for $f \in V_{\rho}$. Obviously,

$$
W_{\rho}\left(f_{0}\right) W_{\rho}\left(f_{1}\right)=\exp \left(i \operatorname{Im}\left\langle f_{0}, f_{1}\right\rangle / 2\right) W_{\rho}\left(f_{0}+f_{1}\right)
$$

is satisfied, if $f_{0}$ and $f_{1}$ belong to a common one-dimensional subspace of $V_{\rho}$. We will show (3) in case $M:=\mathbb{C} f_{0}+\mathbb{C} f_{1}$ is a two-dimensional subspace of $V_{\rho}$. Since the representations $\rho_{N_{0}}^{\prime}$ and $\rho_{N_{1}}^{\prime}$ for $N_{0}:=\mathbb{C} f_{0}$ and $N_{1}:=\mathbb{C} f_{1}$ are nondegenerate, $\rho_{M}^{\prime}$ is nondegenerate, too. Thus there is a representation $\rho_{M}$ of $\mathscr{H}_{M}$ associated with $\rho_{M}^{\prime}$. The same argument as in (i) implies that $f \in M \mapsto \rho_{M}(0, f)$ is a regular representation of the CCR over $M$. So we can show the relation (3) by proving 


$$
W_{\rho}(f)=\rho_{M}(0, f) \quad \text { for } f \in M \text {. }
$$

Obviously $f=0$ satisfies (4); for $f \neq 0$ let $N:=\mathbb{C} f$, and $N^{\perp}$ be the orthogonal complement of $N$ in $M$. For $h \in L^{1}\left(\mathscr{H}_{N}\right)$ and $k \in L^{1}\left(\mathscr{H}_{N^{\perp}}\right)$ we have

$$
\begin{aligned}
& \rho_{N}^{\prime}(h) \rho_{N^{\perp}}^{\prime}(k)=\rho\left(\pi_{N}^{\prime}(h) \pi_{N^{\perp}}^{\prime}(k)\right)= \\
& \rho\left(\int_{\mathbb{R}} \int_{N} \int_{\mathbb{R}} \int_{N^{\perp}} h(t, g) k\left(t^{\perp}, g^{\perp}\right) e^{i\left(t+t^{\perp}\right)} W(g) W\left(g^{\perp}\right) d g^{\perp} d t^{\perp} d g d t\right)= \\
& \rho\left(\int_{\mathbb{R}} \int_{N \times N^{\perp}}\left(\int_{\mathbf{R}} k\left(t^{\perp}, g^{\perp}\right) e^{i t^{\perp}} d t^{\perp}\right) h(t, g) e^{i t} W\left(g+g^{\perp}\right) d g^{\perp} d g d t\right)=\rho_{M}^{\prime}(l),
\end{aligned}
$$

where

$$
l\left(t, g, g^{\perp}\right)=\left(\int_{\mathbb{R}} k\left(t^{\perp}, g^{\perp}\right) e^{i t^{\perp}} d t^{\perp}\right) h(t, g) \quad \text { for } t \in \mathbb{R}, g \in N \text {, and } g^{\perp} \in N^{\perp} .
$$

(Identifying $\mathscr{H}_{M}$ and $\mathbb{R} \times N \times N^{\perp}$, we get $l \in L^{1}\left(\mathscr{H}_{M}\right)$ ). Similarly, we conclude

$$
\begin{aligned}
& \rho_{M}(0, f) \rho_{N}^{\prime}(h) \rho_{N^{\perp}}^{\prime}(k)=\rho_{M}(0, f) \rho_{M}^{\prime}(l)=\rho_{M}^{\prime}\left(_{(0, f)} l\right)= \\
& \rho\left(\int_{\mathbb{R}} \int_{N \times N^{\perp}}\left(\int_{\mathbb{R}} k\left(t^{\perp}, g^{\perp}\right) e^{i t \perp} d t^{\perp}\right)\left(_{(0, f)} h\right)(t, g) e^{i t} W\left(g+g^{\perp}\right) d g^{\perp} d g d t\right)= \\
& \rho_{N}^{\prime}\left(_{(0, f)} h\right) \rho_{N^{\perp}}^{\prime}(k) .
\end{aligned}
$$

Since $\rho_{N^{\perp}}$ is nondegenerate, we get $\rho_{N}(0, f)=\rho_{M}(0, f)$ and equation (4).

Proposition 1.4. Let $\rho: \mathscr{B}(V) \rightarrow \mathscr{L}(K)$ be a factor representation of $\mathscr{B}(V)$ in a Hilbert space $K \neq 0$.

(i) For $N \in \mathcal{N}, \rho_{N}^{\prime}$ is either the zero representation or it is a nondegenerate representation of $L^{1}\left(\mathscr{H}_{N}\right)$.

(ii) $W_{\rho}$ is factorial, too. If $\rho$ is irreducible, $W_{\rho}$ is irreducible, too.

First we show a lemma.

Lemma 1.5. Let $\rho: \mathscr{B}(V) \rightarrow \mathscr{L}(K)$ be a representation of $\mathscr{B}(V)$. For $N \in \mathscr{N}$ the essential space $K_{N}:=\left[\rho_{N}^{\prime}\left(L^{1}\left(\mathscr{H}_{N}\right)\right) K\right]$ of the representation $\rho_{N}^{\prime}$ is an invariant subspace of $\rho$.

Proof. It suffices to show

$$
\rho_{\tilde{N}}^{\prime}(h) \rho_{N}^{\prime}(k) \psi \in K_{N}
$$

for every $\tilde{N} \in \mathcal{N}, \psi \in K, h \in L^{1}\left(\mathscr{H}_{\tilde{N}}\right)$, and $k \in L^{1}\left(\mathscr{H}_{N}\right)$. The case $\tilde{N}=N$ is trivial; otherwise we set $M:=N+\tilde{N}$; by Lemma $1.2 \rho_{\tilde{N}}^{\prime}(h) \rho_{N}^{\prime}(k)$ is in the closure of $\rho_{M}^{\prime}\left(L^{1}\left(\mathscr{H}_{M}\right)\right.$ ). By Lemma 1.2 (using $N$ instead of $N_{0}$ and $\tilde{N}$ instead of $N_{1}$ ) we conclude that $\rho_{\tilde{N}}^{\prime}(h) \cdot \rho_{N}^{\prime}(k)$ belongs to the closure of the linear span of $\rho_{N}^{\prime}\left(L^{1}\left(\mathscr{H}_{N}\right)\right) \cdot \rho_{\tilde{N}}^{\prime}\left(L^{1}\left(\mathscr{H}_{\tilde{N}}\right)\right)$. It follows (5). 
Proof of Proposition 1.4. (i) By Lemma $1.5 K_{N}$ and therefore $\left(K_{N}\right)^{\perp}$ are invariant subspaces of $\rho(\mathscr{B}(V))$. Let $\rho^{\perp}$ be the subrepresentation of $\rho$ defined on $\left(K_{N}\right)^{\perp}$. Let us assume that $\rho_{N}^{\prime}$ is degenerate. Then $\left(K_{N}\right)^{\perp} \neq\{0\}$, and $\rho^{\perp}$ is quasi-equivalent to $\rho$, since $\rho$ is factorial. Thus $\left.\rho^{\perp}\right|_{\pi_{N}^{\prime}\left(L^{1}\left(\mathscr{H}_{N}\right)\right)}=0$ entails $\left.\rho\right|_{\pi_{N}^{\prime}\left(L^{1}\left(\mathscr{H}_{N}\right)\right)}=0$.

(ii) The case $\mathscr{N}_{\rho}=\emptyset$ is trivial. For $\mathscr{N}_{\rho} \neq \emptyset$ from

$$
\begin{array}{r}
\left\{W_{\rho}(f): f \in V_{\rho}\right\}^{\prime}= \\
\bigcap_{N \in \mathcal{N}_{\rho}}\left\{e^{i t} W_{\rho}(f): f \in N, t \in \mathbb{R}\right\}^{\prime}= \\
\bigcap_{N \in \mathcal{N}_{\rho}}\left(\rho_{N}^{\prime}\left(L^{1}\left(\mathscr{H}_{N}\right)\right)\right)^{\prime}=(\text { using (i)) } \\
\bigcap_{N \in \mathcal{N}}\left(\rho_{N}^{\prime}\left(L^{1}\left(\mathscr{H}_{N}\right)\right)\right)^{\prime}=\rho(\mathscr{B}(V))^{\prime},
\end{array}
$$

we get the assertion.

\section{\$2. The Decomposition Theorem}

For the following we need the concept of direct integrals introduced by W.Wils. We recall the definition (see [12]).

Defimition 2.1. Let $(Z, \mu)$ be a measure space; for $\zeta \in Z$ let $H(\zeta)$ be a complex Hilbert space. A linear subspace $\Gamma$ of $\prod_{\zeta \in Z} H(\zeta)$ is called a set of $\mu$ square integrable vector fields, iff

(i) $\zeta \in Z \rightarrow\|\eta(\zeta)\|^{2}$ is $\mu$-integrable for every $\eta=(\eta(\zeta))_{\zeta \in Z} \in \Gamma$.

(ii) If for $\eta \in \prod_{\zeta \in Z} H(\zeta)$ there is an $\eta^{\prime} \in \Gamma$ such that $\eta(\zeta)=\eta^{\prime}(\zeta)$ for almost every $\zeta$, then $\eta \in \Gamma$.

(iii) If $\eta \in \Gamma$ and $h \in L^{\infty}(Z, \mu)$, then $h \cdot \eta=(h(\zeta) \eta(\zeta))_{\zeta \in Z} \in \Gamma$.

(iv) $\Gamma$ with respect to the seminorm $\|\eta\|_{2}=\left(\int_{Z}\|\eta(\zeta)\|^{2} d \mu(\zeta)\right)^{\frac{1}{2}}$ is complete.

The corresponding Hilbert space is called the direct integral of the Hilbert spaces $H(\zeta)$, denoted by

$$
\int_{Z}^{\oplus} H(\zeta) d \mu(\zeta) \text { or } \int_{Z}^{\Gamma} H(\zeta) d \mu(\zeta) .
$$

In order to make a distinction we call the direct integrals defined by the usual concept, which for example is described in Dixmier's books [1] and [2], as "direct integrals in the sense of J.Dixmier". Obviously, these direct integrals also satisfy the Definition 2.1 .

Definition 2.2. Let $H=\int_{Z}^{\Gamma} H(\zeta) d \mu(\zeta)$ be a direct integral of Hilbert 
spaces. Let $W: V \rightarrow \mathscr{U}(H)$ be a regular representation of the CCR over $V$. For $\zeta \in Z$ let $V(\zeta)$ be a complex subspace of $V$, and $W(\zeta): V(\zeta) \rightarrow \mathscr{U}(H(\zeta))$ be a regular representation of the CCR over $V(\zeta)$. Moreover let us assume that for every $f \in V$ there is a $\mu$-negligible set $N_{f}$ such that $f \in V(\zeta)$ for every $\zeta \in Z \backslash N_{f}$, $\int_{Z}^{\Gamma} W(\zeta)(f) d \mu(\zeta)$ is well defined and equal to $W(f)$. (This precisely means that for $\eta=(\eta(\zeta))_{\zeta \in Z} \in \Gamma$ the almost everywhere unique extension $\left(\eta_{f}(\zeta)\right)_{\zeta \in Z}$ of $(W(\zeta)(f) \eta(\zeta))_{\zeta \in Z \backslash N}$ to an element of $\prod_{\zeta \in Z} H(\zeta)$ belongs to $\Gamma$, and that the operator of $\mathscr{L}(H)$, given by

$$
\int_{z}^{\Gamma} \eta(\zeta) d \mu(\zeta) \longrightarrow \int_{z}^{\Gamma} \eta_{f}(\zeta) d \mu(\zeta)
$$

is equal to $W(f)$.)

Then we say:

$$
W=\int_{z}^{\Gamma} W(\zeta) d \mu(\zeta)
$$

is a decomposition of $W$ into regular representations $W(\zeta)$ of the CCR that are defined over subspaces $V(\zeta)$ of $V$.

Now we can formulate the main result of our paper.

Theorem 2.3. Let $W: V \rightarrow \mathscr{U}(H)$ be a cyclic regular representation of the $C C R$ over the complex inner product space $V \neq 0$. Then there exist a regular Borel measure $v$ on a compact space $X$ and a decomposition

$$
W=\int_{X}^{\oplus} W(\varphi) d v(\varphi)
$$

of $W$ into irreducible regular representations $W(\varphi): V(\varphi) \rightarrow \mathscr{U}(H(\varphi))$ of the $C C R$, which are defined over complex subspaces $V(\varphi)$ of $V$.

Supplement. (i) If $V$ is a separable inner product space, almost every $V(\varphi)$ is dense in $V$.

(ii) If the decomposition is constructed as in the proof, the diagonal algebra in $\int_{X}^{\oplus} H(\varphi) d v(\varphi)$ is maximal abelian in $\{W(f): f \in V\}^{\prime}$.

We can formulate this result using the group $\mathscr{H}_{V}$ : Certain representations of the group $\mathscr{H}_{V}$ will be decomposed into certain irreducible representations of subgroups $\mathscr{H}_{V(\varphi)}$ of $\mathscr{H}_{V}$. So we get a decomposition theorem for representations of a non locally compact group, having no equivalent in the theory of locally compact groups.

In the proof we shall have to decompose the identity representation $\mathbb{1}_{\mathscr{B}(V)}$ of 
$\mathscr{B}(V)$. For this purpose we need a theorem due to R.W. Henrichs. First let us give a definition.

Definition 2. Let $\pi$ be a cyclic representation of a $C^{*}$-algebra $\mathscr{A}$ and $\xi$ a cyclic vector for $\pi$ such that $\|\xi\|=1$. We say that a decomposition

$$
\pi=\int_{Z}^{\oplus} \pi(\zeta) d \mu(\zeta)
$$

of $\pi$ is normalized (with respect to $\xi$ ), iff in the corresponding decomposition $\xi$ $=\int_{Z}^{\oplus} \xi(\zeta) d \mu(\zeta)$ of $\xi$ almost every $\xi(\zeta)$ is cyclic for $\pi(\zeta)$ and $\|\xi(\zeta)\|=1$.

Theorem 2.5 ([6]). Let $\pi$ be a cyclic representation of a unital (not necessarily separable) $C^{*}$-algebra $\mathscr{A}$ and $\xi$ a cyclic vector for $\pi$ such that $\|\xi\|$ $=1$. Then there is a (with respect to $\xi$ ) normalized decomposition

$$
\pi=\int_{X}^{\oplus} \pi(\varphi) d \mu(\varphi)
$$

of $\pi$ such that every $\pi(\varphi)$ is an irreducible representation of $\mathscr{A}$, and $\mu$ is a regular Borel measure on the compact space $X$.

Remarks. (i) Let us briefly sketch Henrichs's construction of the decomposition:

Let $\omega$ be the state of $\mathscr{A}$ belonging to $\pi$ and $\xi$, and $\mathscr{C}$ be a maximal abelian von Neumann subalgebra of $\pi(\mathscr{A})^{\prime} . \mu$ is chosen as the orthogonal measure associated with $\omega$ and $\mathscr{C}$. For $\varphi \in \mathscr{S}(\mathscr{A}), \pi(\varphi)$ is the GNS representation of a pure state $\tilde{\varphi} \in \mathscr{S}(\mathscr{A})$ such that

$$
\left\{a \in \mathscr{A}: \varphi\left(a^{*} a\right)=0\right\} \subseteq\left\{a \in \mathscr{A}: \tilde{\varphi}\left(a^{*} a\right)=0\right\} .
$$

The diagonal algebra in the direct integral can be identified with $\mathscr{C}$.

(ii) In [6], §3 R.W. Henrichs varies the decomposition of $\pi$, in which the orthogonal measure associated with $\omega$ and the center $\pi(A)^{\prime} \cap \pi(\mathscr{A})^{\prime \prime}$ of $\pi(\mathscr{A})^{\prime}$ is used, in a similar way in order to get a decomposition of $\pi$ into factorial representations.

If we apply this decomposition for $\mathbb{1}_{\mathscr{B}(V)}$ instead of the decomposition above, we obtain a decomposition of $W$ into factorial regular representations $W(\varphi)$, which are defined over subspaces $V(\varphi)$ of $V$.

Lemmæ 2.6. Let $\xi$ be a cyclic vector for $W\left(\right.$ and therefore for $\left.\mathbb{1}_{\mathscr{B}(V)}\right)$ such that $\|\xi\|=1$. Let

$$
\mathbb{1}_{\mathscr{B}(V)}=\int_{Z}^{\Gamma} \rho(\zeta) d \mu(\zeta)
$$


be a (with respect to $\xi$ ) normalized decomposition of the identical representation $\mathbb{1}_{\mathscr{B}(V)}$ of $\mathscr{B}(V)$ into factorial representations $\rho(\zeta)$. For a fixed $N \in \mathcal{N}, \rho(\zeta)_{N}^{\prime}$ is nondegenerate for almost every $\zeta \in Z$.

Proof. Let $\xi=\int_{Z}^{\Gamma} \xi(\zeta) d \mu(\zeta)$ be the corresponding decomposition of $\xi$; for $\zeta \in Z$ let $\varphi(\zeta)$ be the state of $\mathscr{B}(V)$ belonging to $\rho(\zeta)$ and $\xi(\zeta)$ and $\omega$ be the state of $\mathscr{B}(V)$ belonging to $\mathbb{1}_{\mathscr{B}(V)}$ and $\xi$. For $N \in \mathscr{N}$ let $\left(u_{n}\right)_{n \in \mathbb{N}}$ be an approximate unit in $L^{1}\left(\mathscr{H}_{N}\right)$. Since $\left(\pi_{N}^{\prime}\left(u_{n}\right)\right)_{n \in \mathbb{N}}$ converges strongly to $\mathbb{1}_{H}$, we have

$$
1=\lim _{n \rightarrow \infty} \omega\left(\pi_{N}^{\prime}\left(u_{n}\right)\right)=\lim _{n \rightarrow \infty} \int_{Z} \varphi(\zeta)\left(\pi_{N}^{\prime}\left(u_{n}\right)\right) d \mu(\zeta) .
$$

Since $0 \leq \varphi(\zeta)\left(\pi_{N}^{\prime}\left(u_{n}\right)\right) \leq 1$, it follows $\lim _{n \rightarrow \infty} \varphi(\zeta)\left(\pi_{N}^{\prime}\left(u_{n}\right)\right)=1$ for almost every $\zeta \in \mathbb{Z}$, and Proposition 1.4 (i) implies the assertion.

Remark. Let us consider a decomposition $\pi=\int_{z}^{\oplus} \pi(\zeta) d \mu(\zeta)$ of a nondegenerate representation $\pi$ of a separable $C^{*}$-algebra. If the usual concept of direct integrals is used, then almost every $\pi(\zeta)$ is nondegenerate. For our more general concept of direct integrals such a statement is not correct.

Lemma 2.7。 Let

$$
\mathbb{1}_{\mathscr{B}(V)}=\int_{Z}^{\Gamma} \rho(\zeta) d \mu(\zeta)
$$

be a decomposition of $\mathbb{1}_{\mathscr{B}(V)}$ such that for $\zeta \in Z$ the representation $\rho(\zeta): \mathscr{B}(V)$ $\rightarrow \mathscr{L}(H(\zeta))$ is nondegenerate and for every fixed $N \in \mathscr{N} \rho(\zeta)_{N}^{\prime}$ is nondegenerate almost everywhere. For $\zeta \in Z$, let $V(\zeta):=V_{\rho(\zeta)}$ and $W(\zeta):=W_{\rho(\zeta)}$. Then

$$
W=\int_{Z}^{\Gamma} W(\zeta) d \mu(\zeta)
$$

is a decomposition of $W$ into the regular representations $W(\zeta)$, which are defined over the complex subspaces $V(\zeta)$ of $V$.

Proof. Let $f \in N$, where $N \in \mathcal{N}$. Since $\rho(\zeta)_{N}^{\prime}$ is almost everywhere nondegenerate, $f$ belongs to almost every $V(\zeta)$. Let $\left(u_{n}\right)_{n \in \mathbb{N}}$ be an approximate unit in $L^{1}\left(\mathscr{H}_{N}\right)$ and $v_{n}:={ }_{(0, f)} u_{n}$ for $n \in \mathbb{N}$. Then $\left(\pi_{N}^{\prime}\left(v_{n}\right)\right)_{n \in \mathbb{N}}$ converges strongly to $\pi_{N}(0, f)=W(f)$, and $\left(\rho(\zeta)_{N}^{\prime}\left(v_{n}\right)\right)_{n \in \mathbb{N}}$ to $W(\zeta)(f)$ almost everywhere. Using $\pi_{N}^{\prime}\left(v_{n}\right)$ $=\int_{Z}^{\Gamma} \rho(\zeta)_{N}^{\prime}\left(v_{n}\right) d \mu(\zeta)$ we easily see that $(W(\zeta)(f))_{\zeta \in Z}$ is a measurable, almost everywhere defined field of operators and

$$
W(f)=\int_{z}^{\Gamma} W(\zeta)(f) d \mu(\zeta)
$$


holds.

Proof of Theorem 2.3. The assertion follows from Proposition 1.4, Theorem 2.5 (applied to $\mathbb{1}_{\mathscr{B}(V)}$ ), Lemma 2.6, and Lemma 2.7. It is easy to show Supplement (i); Supplement (ii) follows from Remark (i) after Theorem 2.5.

Remarks 2.8. (i) If $V$ is a Hilbert space, one can get a decomposition of $W$ with the properties of the decomposition of Theorem 2.3, for which, additionally, holds :

All the $V(\varphi)^{\prime} s$ are dense in $\mathbb{V}$.

Let a decomposition $W=\int_{X}^{\oplus} W(\varphi) d \mu(\varphi)$ of $W$ as in Theorem 2.3 be given; for $\varphi \in X$ we define

$$
V(\varphi)^{\perp}:=\{f \in V:\langle f, g\rangle=0 \text { for } g \in V(\varphi)\} .
$$

Let $W(\varphi)^{\perp}: V(\varphi)^{\perp} \rightarrow \mathscr{U}\left(H(\varphi)^{\perp}\right)$ be an irreducible regular representation of the CCR over $V(\varphi)^{\perp}$ (the Fock-representation for example); for $\mathbb{V}(\varphi)^{\perp}=\{0\}$ let $H(\varphi)^{\perp}:=\mathbb{C}$ and $W(\varphi)^{\perp}(0):=\mathbb{1}$.

Let $\quad \tilde{V}(\varphi):=V(\varphi)+V(\varphi)^{\perp}, \quad \tilde{H}(\varphi):=H(\varphi) \otimes H(\varphi)^{\perp}, \quad$ and $\quad \tilde{W}(\varphi): \tilde{\mathbb{V}}(\varphi)$ $\rightarrow \mathscr{U}(\tilde{H}(\varphi))$ such that

$$
\tilde{W}(\varphi)\left(f+f^{\perp}\right)=W(\varphi)(f) \otimes W(\varphi)^{\perp}\left(f^{\perp}\right)
$$

for $f \in V(\varphi)$ and $f^{\perp} \in V(\varphi)^{\perp}$.

$\tilde{V}(\varphi)$ is dense in $V$, and $\tilde{W}(\varphi)$ is an irreducible regular representation of the CCR over $\tilde{V}(\varphi)$. Considering $\tilde{W}(\varphi)$ as extension of $W(\varphi)$, we get the decomposition

$$
W=\int_{X}^{\oplus} \tilde{W}(\varphi) d \mu(\varphi)
$$

of $W$ which has the desired properties.

(ii) In some cases more general spaces $V$ are permitted in the definition of the CCR. We consider real vector spaces $V$, endowed with an antisymmetric bilinear form $\sigma$ such that there is a countable subset $\left\{f_{n}: n \in \mathbb{N}\right\}$ having the following property:

For every $g \in V \backslash\{0\}$ there is an $n \in \mathbb{N}$ such that $\sigma\left(f_{n}, g\right) \neq 0$. (Important examples are real dense subspaces of the separable Hilbert space endowed with the imaginary part of the inner product.) In the definition of the $\mathbb{C C R}$ we then have to replace $\operatorname{Im}\langle f, g\rangle$ by $\sigma(f, g)$.

Using similar methods as above one can get a decomposition theorem for regular representation over such spaces, too (see [10], Theorem 2.2.6). Then the representations of the fibers are irreducible regular representations over 
subspaces $V(\varphi)$ of $V$, for which $\left.\sigma\right|_{V(\varphi) \times V(\varphi)}$ is nondegenerate. (That means that for every $f \in V(\varphi)$ there is a $g \in V(\varphi)$ such that $\sigma(f, g) \neq 0$.)

\section{Chapter 2. The Domains of the Fibers in the Decomposition of Regular Representations of the Canonical Commutation Relations}

\section{§3. Extensions of Direct-Product Representations}

In this Section we construct some irreducible regular representations of the CCR over a dense subspace of the Hilbert space $l^{2}(\mathbb{N})$, which cannot be extended to regular representations over $l^{2}(\mathbb{N})$. For our purposes we need a very general concept of extension; thus the results of the literature (see [11] or [13], for example) are not sufficient.

Defimition 3. 耳. Let $V_{1}$ and $V_{2}$ be real dense subspaces of $l^{2}(\mathbb{N})$ such that $V_{1} \subseteq V_{2}$. Let $W_{1}: V_{1} \rightarrow \mathscr{U}(H)$ and $W_{2}: V_{2} \rightarrow \mathscr{U}(K)$ be regular representations over $V_{1}$ and $V_{2}$, resp., such that $H$ is a closed subspace of $K$ (for the definition of representations of the CCR over real dense subspaces of $l^{2}(\mathbb{N})$ see 2.8 (ii)). Moreover, let $H$ be invariant under $W_{2}$, and let the restriction of $W_{2}$ on $H$ be equal to $W_{1}$. Then we say that $W_{2}$ is an extension of $W_{1}$.

Let $W_{S}: \mathbb{C} \rightarrow \mathscr{U}\left(L^{2}(\mathbb{R})\right)$ be the Schrödinger representation of the CCR over $\mathbb{C}$, defined by

$$
\left(W_{S}(r+i s) f\right)(x)=\exp (\operatorname{ir}(x+s / 2)) f(x+s) .
$$

For $m \in \mathbb{N}$, the $m$-fold tensor product $W_{S}^{m}$ of $W_{S}$ is an irreducible regular representation of the $\mathrm{CCR}$ over $\mathbb{C}^{m}$, called the $m$-dimensional Schrödinger representation.

For $n \in \mathbb{N}$ let $\psi_{n} \in L^{2}(\mathbb{R})_{1}$; moreover, let $\xi:=\bigotimes_{n=1}^{\infty} \psi_{n}$, and let $H_{\xi}$ be the incomplete infinite tensor product of countable many copies of $L^{2}(\mathbb{R})$, distinguished by $\xi$ (see [8] or [3]). Furthermore, for $n \in \mathbb{N}$ let $e_{n}:=\left(\delta_{j n}\right)_{j \in \mathbb{N}} \in l^{2}(\mathbb{N})$ and $V_{0}$ be the complex algebraic span of the $e_{n}, n \in \mathbb{N}$. By putting

$$
W_{\xi}^{0}\left(\sum_{n=1}^{m} \lambda_{n} e_{n}\right) \bigotimes_{n=1}^{\infty} \varrho_{n}:=\bigotimes_{n=1}^{m} W_{S}\left(\lambda_{n}\right) \varrho_{n} \otimes \bigotimes_{n=m+1}^{\infty} \varrho_{n},
$$

we define an irreducible regular representation $W_{\xi}^{0}: V_{0} \rightarrow \mathscr{U}\left(H_{\xi}\right)$ of the CCR over $V_{0}$. It is called the direct-product representation distinguished by $\xi$ (see [7]).

Let us recall a result of L. Streit (see $[11], \S 4)$ concerning extensions of $W_{\xi}^{0}$. Consider the elements $\sum_{n=1}^{\infty} \lambda_{n} e_{n}$ of $l^{2}(\mathbb{N})$ such that for every $t \in \mathbb{R}$ $\otimes_{n=1}^{\infty} W_{S}\left(t \lambda_{n}\right) \psi_{n}$ belongs to $H_{\xi}$, that means that

$$
\sum_{n=1}^{\infty}\left|\left\langle W_{S}\left(t \lambda_{n}\right) \psi_{n}, \psi_{n}\right\rangle-1\right|<\infty
$$


for every $t \in \mathbb{R}$. They form a real subspace $V_{\xi}$ of $l^{2}(\mathbb{N})$. Obviously we have $V_{\xi} \supseteq V_{0}$. By defining

$$
W_{\xi}\left(\sum_{n=1}^{\infty} \lambda_{n} e_{n}\right) \bigotimes_{n=1}^{\infty} \varrho_{n}=\bigotimes_{n=1}^{\infty} W_{S}\left(\lambda_{n}\right) \varrho_{n} \quad \text { for } \bigotimes_{n=1}^{\infty} \varrho_{n} \in H_{\xi} \text { and } \sum_{n=1}^{\infty} \lambda_{n} e_{n} \in V_{\xi},
$$

we get an irreducible regular representation $W_{\xi}: V_{\xi} \rightarrow \mathscr{U}\left(H_{\xi}\right)$ of the CCR over $V_{\xi} . \quad W_{\xi}$ is an extension of $W_{\xi}^{0}$.

The following result makes it possible to find direct-product representations having no extension to regular representations of the CCR over $l^{2}(\mathbb{N})$.

Theorem 3.2. Let $g=\sum_{n=1}^{\infty} \mu_{n} e_{n} \in l^{2}(\mathbb{N}) \backslash V_{\xi}$ such that for every $t \in \mathbb{R} \backslash\{0\}$ $\bigotimes_{n=1}^{\infty} W_{S}\left(t \mu_{n}\right) \psi_{n}$ is not weakly equivalent to $\bigotimes_{n=1}^{\infty} \psi_{n}$, that means that

$$
\sum_{n=1}^{\infty}||\left\langle W_{S}\left(t \mu_{n}\right) \psi_{n}, \psi_{n}\right\rangle|-1|=\infty
$$

for every $t \in \mathbb{R} \backslash\{0\}$. Then $W_{\xi}^{0}$ cannot be extended to a regular representation of the CCR over a real subspace $V$ of $l^{2}(\mathbb{N})$, containing $g$ and $V_{0}$.

So, if such a $g$ exists, $W_{\xi}$ and $W_{\xi}^{0}$ have no extension to a regular representation over $l^{2}(\mathbb{N})$.

First let us show a lemma.

Lemma 3.3. Let $\omega_{\xi}^{0}$ be the pure state of the CCR algebra $\mathscr{A}\left(\mathbb{V}_{0}\right)$ belonging to $W_{\xi}^{0}$ and the cyclic vector $\xi=\otimes_{n=1}^{\infty} \psi_{n}$. Moreover, let $V$ be a real subspace of $l^{2}(\mathbb{N})$ such that $V \supseteq V_{0}$, and let $\omega$ be a state of $\mathscr{A}(V)$, extending $\omega_{\xi}^{0}$ such that the corresponding representation $W_{\omega}$ of the $C C R$ over $V$ is regular. Then for $f$ $=\sum_{n=1}^{\infty} \lambda_{n} e_{n} \in \mathbb{V}$

$$
|\omega(w(f))| \leq \prod_{n=1}^{\infty}\left|\left\langle W_{S}\left(\lambda_{n}\right) \psi_{n}, \psi_{n}\right\rangle\right|
$$

holds.

Proof. We show

$$
|\omega(w(f))| \leq \prod_{n=1}^{m}\left|\left\langle W_{S}\left(\lambda_{n}\right) \psi_{n}, \psi_{n}\right\rangle\right|
$$

for every $m \in \mathbb{N}$.

Let $m$ be fixed. We identify $\mathbb{C}^{m}$ and the subspace $\left\{\sum_{n=1}^{m} \lambda_{n} e_{n}: \lambda_{1}, \ldots, \lambda_{m} \in \mathbb{C}\right\}$ of $V_{0}$ in a canonical way.

Let $\left(W_{\omega}, H_{\omega}, \xi_{\omega}\right)$ be the GNS representation of the $\mathbb{C C R}$ over $V$ corresponding to $\omega$. (Instead of the representation $\pi_{\omega}$ of $\mathscr{A}(V)$ belonging to $\omega$ we apply the representation $W_{\omega}$ of the CCR over $V$ that is associated with $\pi_{\omega}$.) By the theorem of Stone and von Neumann the restriction $\left.W_{\omega}\right|_{\mathbb{C}^{m}}$ of $W_{\omega}$ to a 
representation over $\mathbf{C}^{m}$ is a multiple of the Schrödinger representation $W_{S}^{m}$. Therefore, we may assume that

$$
H_{\omega}=\bigotimes_{n=1}^{m} L^{2}(\mathbf{R}) \otimes l^{2}(I)
$$

where $I$ is a suitable index set, and

$$
W_{\omega}\left(\sum_{n=1}^{m} \lambda_{n} e_{n}\right)=\bigotimes_{n=1}^{m} W_{S}\left(\lambda_{n}\right) \otimes 1
$$

for $\lambda_{1}, \ldots, \lambda_{m} \in \mathbf{C}$

Next we need more information about $\xi_{\omega}$. Let $\omega_{m}$ be the restriction of $\omega$ to $\mathscr{A}\left(\mathbb{C}^{m}\right)$. From

$$
\omega_{m}\left(w\left(\sum_{n=1}^{m} \lambda_{n} e_{n}\right)\right)=\prod_{n=1}^{m}\left\langle W_{S}\left(\lambda_{n}\right) \psi_{n}, \psi_{n}\right\rangle=\left\langle\left(\bigotimes_{n=1}^{m} W_{S}\left(\lambda_{n}\right)\right) \bigotimes_{n=1}^{m} \psi_{n}, \bigotimes_{n=1}^{m} \psi_{n}\right\rangle
$$

for $\lambda_{1}, \ldots, \lambda_{m} \in \mathbf{C}$ we conclude that the GNS-representation for $\omega_{m}$ is equal to

$$
\left(W_{S}^{m}, \bigotimes_{n=1}^{m} L^{2}(\mathbf{R}), \bigotimes_{n=1}^{m} \psi_{n}\right) \text {. }
$$

So $\omega_{m}$ is a pure state.

Let $\left(\eta_{t}\right)_{l \in I}$ be an orthonormal base of $l^{2}(I)$. We can write $\xi_{\omega}$ as the sum $\sum_{l \in I} \varrho_{l} \otimes \eta_{l}$ where $\varrho_{l} \in \bigotimes_{n=1}^{m} L^{2}(\mathbf{R})$ and $\sum_{l \in I}\left\|\varrho_{l}\right\|^{2}=1$. For $g \in \mathbf{C}^{m}$ we have

$$
\begin{aligned}
\omega_{m}(w(g)) & =\omega(w(g))=\left\langle\left(W_{S}^{m}(g) \otimes 1\right)\left(\sum_{i \in I} \varrho_{l} \otimes \eta_{l}\right),\left(\sum_{l \in I} \varrho_{l} \otimes \eta_{l}\right)\right\rangle= \\
& =\sum_{l \in I}\left\langle W_{S}^{m}(g) \varrho_{l}, \varrho_{l}\right\rangle=\sum_{l \in I} \omega^{l}(w(g)),
\end{aligned}
$$

where $\omega^{l}$ is the positive linear form of $\mathscr{A}\left(\mathbf{C}^{m}\right)$ determined by $\omega^{l}(w(g))=\left\langle W_{S}^{m}(g) \varrho_{l}\right.$, $\varrho_{l}>$ for $g \in \mathbf{C}^{m}$. Since $\omega_{m}$ is pure, we have $\omega^{l}=t_{l}^{2} \omega_{m}$, where $t_{l}$ is a suitable nonnegative number. From (6) we see that there is a unitary operator $U$ in $\bigotimes_{n=1}^{m} L^{2}(\mathbf{R})$ such that

$$
U \varrho_{l}=t_{l} \bigotimes_{n=1}^{m} \psi_{n} \text { and } U W_{S}^{m}(g) U^{*}=W_{S}^{m}(g) \quad \text { for } g \in \mathbf{C}^{m} \text {. }
$$

Since $W_{S}^{m}$ is irreducible, $U=\gamma 1$, where $|\gamma|=1$, and $\varrho_{l}=\alpha_{l} \otimes_{n=1}^{m} \psi_{n}$, where $\alpha_{l}$ $=t_{l} \bar{\gamma}$. We get

$$
\xi_{\omega}=\bigotimes_{n=1}^{m} \psi_{n} \otimes \eta \quad \text { where } \eta:=\sum_{i \in I} \alpha_{l} \eta_{l} .
$$

Moreover, for $f=\sum_{n=1}^{\infty} \lambda_{n} e_{n} \in V$ we get 


$$
W_{\omega}\left(\sum_{n=m+1}^{\infty} \lambda_{n} e_{n}\right) \in\left\{W_{S}^{m}(g) \otimes \mathbb{1}: g \in \mathbb{C}^{m}\right\}^{\prime}=\mathbb{C} \mathbb{1} \otimes \mathscr{L}\left(l^{2}(I)\right)
$$

therefore we have

$$
W_{\omega}\left(\sum_{n=m+1}^{\infty} \lambda_{n} e_{n}\right)=\mathbb{1} \otimes W_{I}\left(\sum_{n=m+1}^{\infty} \lambda_{n} e_{n}\right)
$$

where $W_{I}\left(\sum_{n=m+1}^{\infty} \lambda_{n} e_{n}\right)$ is a unitary operator in $\mathscr{L}\left(l^{2}(I)\right)$.

It follows

$$
\begin{aligned}
W_{\omega}\left(\sum_{n=1}^{\infty} \lambda_{n} e_{n}\right) & =W_{\omega}\left(\sum_{n=1}^{m} \lambda_{n} e_{n}\right) \cdot W_{\omega}\left(\sum_{n=m+1}^{\infty} \lambda_{n} e_{n}\right) \\
& =W_{S}^{m}\left(\sum_{n=1}^{m} \lambda_{n} e_{n}\right) \otimes W_{I}\left(\sum_{n=m+1}^{\infty} \lambda_{n} e_{n}\right) .
\end{aligned}
$$

(7) and $\|\eta\|=1$ finally yield

$$
\begin{aligned}
\mid \omega(w(f)) & =\left|\left\langle\left(\bigotimes_{n=1}^{m} W_{S}\left(\lambda_{n}\right) \otimes W_{I}\left(\sum_{n=m+1}^{\infty} \lambda_{n} e_{n}\right)\right)\left(\bigotimes_{n=1}^{m} \psi_{n} \otimes \eta\right),\left(\bigotimes_{n=1}^{m} \psi_{n} \otimes \eta\right)\right\rangle\right| \\
& \leq \prod_{n=1}^{m}\left|\left\langle W_{S}\left(\lambda_{n}\right) \psi_{n}, \psi_{n}\right\rangle\right| \cdot 1 .
\end{aligned}
$$

Proof of Theorem 3.2. Let us assume that $W: V \rightarrow \mathscr{U}(K)$ is a regular extension of $W_{\xi}^{0}$. Let $\omega$ be the state of $\mathscr{A}(V)$ corresponding to $W$ and $\xi$ (considered as an element of $K$ ). By assumption,

$$
\prod_{n=1}^{\infty}\left|\left\langle W_{S}\left(t \mu_{n}\right) \psi_{n}, \psi_{n}\right\rangle\right|=0
$$

holds for every $t \in \mathbb{R} \backslash\{0\}$. Lemma 3.3 implies $\omega(w(t g))=0$ for $t \in \mathbb{R} \backslash\{0\}$. This is a contradiction to the regularity of $W$, from which $\lim _{t \rightarrow 0} \omega(w(t g))=1$ follows.

.

Now we discuss some examples. First let us introduce some notation, which is used in the next section, too.

$$
\begin{aligned}
& \phi_{0} \in L^{2}(\mathbb{R}), \text { defined by } \phi_{0}(x)=\frac{\sqrt{2}}{\pi^{1 / 4}} x \exp \left(-\frac{1}{2} x^{2}\right) \text { for } x \in \mathbb{R} \\
& \phi_{1} \in L^{2}(\mathbb{R}) \text {, defined by } \phi_{1}(x)=\frac{1}{\sqrt{2}} \chi_{[-1,1]}(x) \text { for } x \in \mathbb{R} \text {, and } \\
& e_{i}(\lambda):=\left\langle W_{S}(\lambda) \phi_{i}, \phi_{i}\right\rangle \text { for } \lambda \in \mathbb{C} \text { and } i=0,1 .
\end{aligned}
$$

Corollary 3.4. Let $\mathbb{N}=N_{0} \cup N_{1}$ be a decomposition of $\mathbb{N}, \beta(n)=0$ for $n \in N_{0}$, and $\beta(n)=1$ for $n \in N_{1}$. For $\xi:=\bigotimes_{n=1}^{\infty} \phi_{\beta(n)}$ we have 


$$
V_{\xi}=\left\{\sum_{n=1}^{\infty} \lambda_{n} e_{n} \in l^{2}(\mathbb{N}): \sum_{n \in N_{1}}\left|\operatorname{Im} \lambda_{n}\right|<\infty\right\} .
$$

If $V \supseteq V_{0}$ is a subspace of $l^{2}(\mathbb{N})$ not contained in $V_{\xi}, W_{\xi}^{0}$ cannot be extended to a regular representation over $V$.

Proof. It is easy to calculate that

$$
e_{1}(\lambda)=1-\frac{|\operatorname{Im} \lambda|}{2}-\frac{(\operatorname{Re} \lambda)^{2}}{6}+o\left(|\lambda|^{2}\right)
$$

for $|\lambda| \leq 2$. It follows that for $\sum_{n=1}^{\infty} \lambda_{n} e_{n} \in l^{2}(\mathbb{N})$

$$
\sum_{n=1}^{\infty}\left|e_{1}\left(\lambda_{n}\right)-1\right|<\infty \Longleftrightarrow \sum_{n=1}^{\infty}\left|\operatorname{Im} \lambda_{n}\right|<\infty
$$

holds. From

$$
e_{0}(\lambda)=\left(1-\frac{|\lambda|^{2}}{2}\right) \exp \left(-\frac{1}{4}|\lambda|^{2}\right)
$$

we see that

$$
\sum_{n=1}^{\infty}\left|e_{0}\left(\lambda_{n}\right)-1\right|<\infty
$$

is satisfied for every $\sum_{n=1}^{\infty} \lambda_{n} e_{n} \in l^{2}(\mathbb{N})$. This shows the equation for $V_{\xi}$; applying Theorem 3.2 and observing that $e_{0}(\lambda) \geq 0$ and $e_{1}(\lambda) \geq 0$ if $\lambda$ has a sufficiently small modulus, we get the assertion.

\section{§4. An Example, Part 1}

In this Section we form the direct integral of irreducible regular representations of the CCR, which are defined over proper subspaces of $l^{2}(\mathbb{N})$ and which cannot be extended to regular representations over $l^{2}(\mathbb{N})$, and get a cyclic regular representation $W_{Y}$ of the $\mathrm{CCR}$ over $l^{2}(\mathbb{N})$.

\section{Notation.}

$Y:=\{0,1\}^{\mathbb{N}}=\left\{\left(\alpha_{n}\right)_{n \in \mathbb{N}}: \alpha_{n} \in\{0,1\}\right.$ for $\left.n \in \mathbb{N}\right\}$ is a metrizable compact abelian group; let $\mu_{Y}$ be the normalized Haar measure of $Y$.

In this Section we identify $\mathbb{N}$ with the disjoint union $\bigcup_{j=1}^{\infty}\{0,1\}^{j}$ by using an arbitrary but fixed bijection.

Let us introduce some further notation used in the following. Let $k$ be an element of $\mathbb{N}$.

$$
\alpha^{k}:=\left(\alpha_{1}, \ldots, \alpha_{k}\right) \in\{0,1\}^{k}(\subseteq \mathbb{N}) \quad \text { for } \alpha=\left(\alpha_{n}\right)_{n \in \mathbb{N}} \in Y,
$$




$$
\begin{array}{ll}
l^{k}:=\left(l_{1}, \ldots, l_{k}\right) \in\{0,1\}^{k}(\subseteq \mathbf{N}) & \text { for } j \geq k \text { and } l=\left(l_{1}, \ldots, l_{j}\right) \in\{0,1\}^{j}, \\
I_{k}:=\bigcup_{j=1}^{k}\{0,1\}^{j}(\subseteq \mathbb{N}), & \text { for } n \in\{0,1\}^{k}, \\
I_{k}^{n}:=I_{k} \backslash\left\{n^{1}, \ldots, n^{k}\right\} & \text { for } n \in\{0,1\}^{k}, \\
\mathbf{N}_{k}:=\mathbb{N} \backslash\{0,1\}^{k}, & \text { for } j \geq k \text { and } n \in\{0,1\}^{k}, \\
C_{n}^{(k)}:=\left\{\alpha \in Y: \alpha^{k}=n\right\} & \text { for } \alpha \in Y \text { and } m \in \mathbb{N}, \\
G_{n, j}^{(k)}:=\left\{l \in\{0,1\}^{j}: l^{k}=n\right\} & \text { for } m, n \in\{0,1\}^{k}, \\
\delta(m, \alpha):=\left\{\begin{array}{l}
1, \text { if } m=\alpha^{k} \text { for a suitable } k \in \mathbb{N} \\
0 \text { otherwise }
\end{array}\right. & \\
\delta(m, n):=\left\{\begin{array}{l}
1 \text { for } m=n \\
0 \text { otherwise }
\end{array}\right. & \text { for } \alpha \in Y\left(\text { Definition of } \phi_{0} \text { and } \phi_{1} \text { before } 3.4\right), \\
\xi_{\alpha}:=\bigotimes_{n=1}^{\infty} \phi_{\delta(n, \alpha)} & \\
W_{\alpha}:=W_{\xi_{\alpha}}, W_{\alpha}^{0}:=W_{\xi_{\alpha}}^{0}, V_{\alpha}:=V_{\xi_{\alpha}}, H_{\alpha}:=H_{\xi_{\alpha}} .
\end{array}
$$

$\S 3$ implies that for $\alpha \in Y \quad W_{\alpha}: V_{\alpha} \rightarrow \mathscr{U}\left(H_{\alpha}\right)$ is an irreducible regular representation of the CCR over

$$
V_{\alpha}=\left\{\sum_{n=1}^{\infty} \lambda_{n} e_{n} \in l^{2}(\mathbb{N}): \sum_{j=1}^{\infty}\left|\operatorname{Im} \lambda_{\alpha \jmath}\right|<\infty\right\},
$$

and there is no regular representation of the CCR over $l^{2}(\mathbb{N})$ extending $W_{\alpha}$.

Lemma 4.1. For every $f \in l^{2}(\mathbb{N}) N_{f}:=\left\{\alpha \in Y: f \notin V_{\alpha}\right\}$ is a $\mu_{Y}$-negligible set.

Proof. $\quad N_{f}=\emptyset$ for $f=0$; from now on let $f=\sum_{n=1}^{\infty} \lambda_{n} e_{n} \neq 0$. For $k \in \mathbb{N}$ we have

$$
N_{f}=\left\{\alpha \in Y: \sum_{j=1}^{\infty}\left|\operatorname{Im} \lambda_{\alpha \jmath}\right|=\infty\right\} \subseteq \bigcup_{j=k}^{\infty} M_{j},
$$

where $M_{j}:=\left\{\alpha \in Y:\left|\lambda_{\alpha^{J}}\right| \geq\|f\| / j^{2}\right\}$. It follows

$$
N_{f} \subseteq \bigcap_{k=1}^{\infty}\left(\bigcup_{j=k}^{\infty} M_{j}\right)
$$

It is

$$
M_{j}=\bigcup_{n \in J_{j}} C_{n}^{(j)} \text { where } J_{j}:=\left\{n \in\{0,1\}^{j}:\left|\lambda_{n}\right| \geq \frac{\|f\|}{j^{2}}\right\} .
$$

Therefore, $\mu_{Y}\left(M_{j}\right)=\left|J_{j}\right| \cdot 1 / 2^{j}$. From 


$$
\frac{\left|J_{j}\right| \cdot\|f\|^{2}}{j^{4}} \leq \sum_{n \in J_{j}}\left|\lambda_{n}\right|^{2} \leq\|f\|^{2}
$$

we get $\left|J_{j}\right| \leq j^{4}$ and $\mu_{Y}\left(M_{j}\right) \leq j^{4} / 2^{j}$. This implies

$$
\mu_{Y}\left(\bigcap_{k=1}^{\infty}\left(\bigcup_{j=k}^{\infty} M_{j}\right)\right)=\inf _{k \in \mathbb{N}} \mu_{Y}\left(\bigcup_{j=k}^{\infty} M_{j}\right) \leq \inf _{k \in \mathbb{N}} \sum_{j=k}^{\infty} \frac{j^{4}}{2^{j}}=0 ;
$$

from (8) we receive the assertion.

We put

$$
H_{Y}:=\int_{Y}^{\oplus} H_{\alpha} d \alpha
$$

where a direct integral in the sense of $J$. Dixmier is used. For $f \in V_{0}$, $\left(W_{\alpha}(f)\right)_{\alpha \in Y}$ is a measurable field of operators. For $f=\sum_{n=1}^{\infty} \lambda_{n} e_{n} \in l^{2}(\mathbf{N})$ and $\alpha \in Y \backslash N_{f},\left(W_{\alpha}\left(\sum_{n=1}^{m} \lambda_{n} e_{n}\right)\right)_{m \in \mathbf{N}}$ converges strongly to $W_{\alpha}(f)($ see [11], Lemma 5); so $\left(W_{\alpha}(f)\right)_{\alpha \in Y}$ is an almost everywhere defined measurable field of operators, and by

$$
W_{Y}: l^{2}(\mathbb{N}) \longrightarrow \mathscr{U}\left(H_{Y}\right), W_{Y}(f)=\int_{Y}^{\oplus} W_{\alpha}(f) d \alpha,
$$

a regular representation of the CCR over $l^{2}(\mathbf{N})$ is given.

Let $W_{Y}^{0}$ be the restriction of $W_{Y}$ to $V_{0}$.

Proposition 4.2. (i) $\xi_{Y}:=\int_{Y}^{\oplus} \xi_{\alpha} d \alpha$ is a cyclic vector for $W_{Y}^{0}$ and $W_{Y}$.

(ii) For the diagonal algebra $\mathscr{D}$ in $H_{Y}=\int_{Y}^{\oplus} H_{\alpha} d \alpha$,

$$
\mathscr{D}=\left\{W_{Y}(f): f \in l^{2}(\mathbf{N})\right\}^{\prime}=\left\{W_{Y}^{0}(f): f \in V_{0}\right\}^{\prime}
$$

holds.

We denote the state of $\mathscr{A}\left(l^{2}(\mathbb{N})\right)\left(\mathscr{A}\left(V_{0}\right)\right.$, resp.), corresponding to $W_{Y}\left(W_{Y}^{0}\right.$, resp.) and $\xi_{Y}$, by $\omega_{Y}\left(\omega_{Y}^{0}\right.$, resp.).

Proof. (i) Since $\int_{Y}^{\oplus} \xi_{\alpha} d \alpha$ is a separating vector for $\mathscr{D}$, (i) follows from (ii).

(ii) In several steps:

(a) It suffices to show

$$
\int_{Y}^{\oplus} \chi_{C_{m}^{(k)}}(\alpha) 1 d \alpha \in\left\{W_{Y}^{0}(f): f \in V_{0}\right\}^{\prime \prime}
$$

for $k \in \mathbb{N}$ and $m \in\{0,1\}^{k}$. 
For: Since $\mathscr{D}$ is generated by

$$
\left\{\int_{Y}^{\oplus} \chi_{C_{m}^{(k)}}(\alpha) \mathbb{1} d \alpha: k \in \mathbb{N}, m \in\{0,1\}^{k}\right\},
$$

$\mathscr{D} \subseteq\left\{W_{Y}^{0}(f): f \in V_{0}\right\}^{\prime \prime}$ is satisfied. Since $\mathscr{D} \subseteq\left\{W_{Y}^{0}(f): f \in V_{0}\right\}^{\prime}$, we get

$$
\mathscr{D} \subseteq\left\{W_{Y}^{0}(f): f \in V_{0}\right\}^{\prime} \cap\left\{W_{Y}^{0}(f): f \in V_{0}\right\}^{\prime \prime} .
$$

Since in the decomposition $W_{Y}^{0}=\int_{Y}^{\oplus} W_{\alpha}^{0} d \alpha$ of $W_{Y}^{0}$ the fibers $W_{\alpha}^{0}$ are irreducible and regular, the diagonal algebra $\mathscr{D}$ is a maximal abelian von Neumann subalgebra of $\left\{W_{Y}^{0}(f): f \in V_{0}\right\}^{\prime}$ (see [9], Lemma 1.2). Now it is easy to conclude that

$$
\mathscr{D}=\left\{W_{Y}^{0}(f): f \in V_{0}\right\}^{\prime} \cap\left\{W_{Y}^{0}(f): f \in V_{0}\right\}^{\prime \prime}=\left\{W_{Y}^{0}(f): f \in \mathbb{V}_{0}\right\}^{\prime} .
$$

The considerations after the proof of Lemma 4.1 establish that for $f$ $=\sum_{n=1}^{\infty} \lambda_{n} e_{n} \in l^{2}(\mathbb{N})\left(W_{Y}\left(\sum_{n=1}^{m} \lambda_{n} e_{n}\right)\right)_{m \in \mathbb{N}}$ converges strongly to $W_{Y}(f)$, and thus

$$
\mathscr{D}=\left\{W_{Y}^{0}(f): f \in V_{0}\right\}^{\prime}=\left\{W_{Y}(f): f \in l^{2}(\mathbb{N})\right\}^{\prime}
$$

holds.

(b) Let $j \in \mathbb{N}$ be fixed. For $\alpha \in Y$ let $H_{\alpha, j}$ be the incomplete tensor product $\otimes_{n \in \mathbb{N}_{J}} L^{2}(\mathbb{R})$ distinguished by $\otimes_{n \in \mathbb{N}_{J}} \phi_{\delta(n, \alpha)} ;$ moreover, let $H_{Y, j}:=\int_{Y}^{\oplus} H_{\alpha, j} d \alpha$ and $L_{j}:=\bigotimes_{n \in\{0,1\},} L^{2}(\mathbb{R})$.

Applying [1], Proposition 11, p. 175, we find that there is a unique Hilbert space isomorphism

$$
U: L_{j} \otimes H_{Y, j} \rightarrow H_{Y}
$$

which maps

$$
\psi \otimes \int_{Y}^{\oplus} \eta(\alpha) d \alpha \text { into } \int_{Y}^{\oplus} \psi \otimes \eta(\alpha) d \alpha
$$

for $\psi \in L_{j}$ and $\int_{Y}^{\oplus} \eta(\alpha) d \alpha \in H_{Y, j}$. (Identifying $L_{j} \otimes H_{\alpha, j}$ and $H_{\alpha}$, we consider $\psi \otimes \eta(\alpha)$ as an element of $H_{\alpha}$.)

Let $V_{j}$ be the subspace

$$
\left\{\sum_{n \in \mathbb{N}_{j}} \lambda_{n} e_{n} \in l^{2}(\mathbb{N}): \lambda_{n}=0 \text { for almost every } n \in \mathbb{N}_{j}\right\}
$$

of $V_{0}$. For $\alpha \in Y$ we define a regular representation $W_{\alpha, j}^{0}: V_{j} \rightarrow \mathscr{U}\left(H_{\alpha, j}\right)$ of the CCR over $V_{j}$ by 


$$
W_{\alpha, j}^{0}\left(\sum_{n \in \mathbf{N}_{j}} \lambda_{n} e_{n}\right)=\bigotimes_{n \in \mathbb{N}_{j}} W_{S}\left(\lambda_{n}\right)
$$

Then by putting

$$
W_{Y, j}^{0}\left(\sum_{n=1}^{\infty} \lambda_{n} e_{n}\right)=\bigotimes_{n \in\{0,1\}^{\jmath}} W_{S}\left(\lambda_{n}\right) \otimes \int_{Y}^{\oplus} W_{\alpha, j}^{0}\left(\sum_{n \in \mathbb{N}_{j}} \lambda_{n} e_{n}\right) d \alpha
$$

for $\sum_{n=1}^{\infty} \lambda_{n} e_{n} \in V_{0}$, we obtain a regular representation

$$
W_{Y, j}^{0}: V_{0} \rightarrow \mathscr{U}\left(L_{j} \otimes H_{Y, j}\right)
$$

of the CCR over $V_{0}$, which is transformed by $U$ into $W_{Y}^{0}$.

(c) For $j \in \mathbb{N}$ and $l \in\{0,1\}^{j}$ let

$$
\psi_{l}:=\bigotimes_{n \in\{0,1\}^{j}} \phi_{\delta(n, l)} \in L_{j} .
$$

From now on, let $k \in \mathbb{N}$ be fixed.

For $m \in\{0,1\}^{k}$ and $j \geq k$ let $P_{m j} \in \mathscr{L}\left(L_{j}\right)$ denote the orthogonal projection onto

$$
\left[\psi_{l}: l \in\{0,1\}^{j}, l^{k}=m\right] ;
$$

moreover, let $Q_{m j}:=U\left(P_{m j} \otimes \mathbb{1}_{H_{Y,}}\right) U^{*}$.

Since the Schrödinger representation $W_{S}$ is irreducible,

$$
\mathscr{L}\left(L_{j}\right)=\left\{\bigotimes_{n \in\{0,1\}^{3}} W_{S}\left(\lambda_{n}\right): \lambda_{n} \in \mathbb{C} \text { for } n \in\{0,1\}^{j}\right\}^{\prime \prime}
$$

it follows

$$
P_{m j} \otimes \mathbb{1} \in\left\{\bigotimes_{n \in\{0,1\}} W_{S}\left(\lambda_{n}\right): \lambda_{n} \in \mathbb{C} \text { for } n \in\{0,1\}^{j}\right\}^{\prime \prime} \otimes \mathbb{C} \mathbb{1} \subseteq\left\{W_{Y, j}^{0}(f): f \in V_{0}\right\}^{\prime \prime}
$$

and $Q_{m j} \in\left\{W_{Y}^{0}(f): f \in V_{0}\right\}^{\prime \prime}$ for $j \geq k$. Now we can prove (9) by showing that $\left(Q_{m j}\right)_{j \geq k}$ converges strongly to $\int_{Y}^{\oplus} \chi_{C_{m}^{(k)}}(\alpha) \mathbb{1} d \alpha$ and by applying the density theorem due to J. von Neumann.

(d) Since $\sup _{j \geq k}\left\|Q_{m j}\right\|=1<\infty$, it suffices to show

$$
\lim _{\substack{j \rightarrow \infty \\ j \geq k}} Q_{m j} \eta=\int_{Y}^{\oplus} \chi_{C_{m}^{(k)}}(\alpha) \mathbb{1} d \alpha \cdot \eta
$$

only for those $\eta$ 's that belong to a suitable generating set of the Hilbert space $H_{Y}$. Therefore we only consider the case 


$$
\eta=\int_{Y}^{\oplus} h(\alpha) \bigotimes_{n=1}^{\infty} \varrho_{n}(\alpha) d \alpha,
$$

where $h \in L^{\infty}(Y)$ and a $j_{0} \geq k$ exists such that $\varrho_{n}(\alpha)=\phi_{\delta(n, \alpha)}$ for $j \geq j_{0}, n \in\{0,1\}^{j}$, and $\alpha \in Y$ (that means that $\otimes_{n \in\{0,1\}} \varrho_{n}(\alpha)=\psi_{l}$ for $j \geq j_{0}, \quad l \in\{0,1\}^{j}$, and $\left.\alpha \in C_{l}^{(j)}\right)$. For $j \geq j_{0}$ we get

$$
\begin{aligned}
& Q_{m j} \eta=\left(\text { identifying } H_{\alpha} \text { and } L_{j} \otimes H_{\alpha, j}\right. \text { ) } \\
& =Q_{m j} \int_{Y}^{\oplus} \sum_{l \in\{0,1\},} \chi_{C_{l}^{(J)}}(\alpha) h(\alpha) \psi_{l} \otimes \bigotimes_{n \in \mathbb{N}_{j}} \varrho_{n}(\alpha) d \alpha= \\
& =Q_{m j} U^{*} \sum_{l \in\{0,1\},} \psi_{l} \otimes \int_{Y}^{\oplus} \chi_{C_{l}^{(J)}}(\alpha) h(\alpha) \bigotimes_{n \in \mathbb{N}_{j}} \varrho_{n}(\alpha) d \alpha= \\
& =U \sum_{l \in\{0,1\}^{\jmath}} P_{m j} \psi_{l} \otimes \int_{Y}^{\oplus} \chi_{C_{l}^{(J)}}(\alpha) h(\alpha) \bigotimes_{n \in \mathbb{N}_{j}} \varrho_{n}(\alpha) d \alpha=(*) \\
& =U \sum_{l \in G_{m, J}^{(k)}} \psi_{l} \otimes \int_{Y}^{\oplus} \chi_{C_{l}^{(J)}}(\alpha) h(\alpha) \otimes_{n \in \mathbb{N}_{J}} \varrho_{n}(\alpha) d \alpha= \\
& =\int_{Y}^{\oplus} \sum_{l \in G_{m, J}^{(k)}} \chi_{C_{l}^{(J)}}(\alpha) h(\alpha) \bigotimes_{n=1}^{\infty} \varrho_{n}(\alpha) d \alpha=\int_{Y}^{\oplus} \chi_{C_{m}^{(k)}}(\alpha) \mathbb{1} d \alpha \cdot \eta ;
\end{aligned}
$$

in $(*)$ we used the fact that $\left\langle\phi_{1}, \phi_{2}\right\rangle=0$, and therefore $P_{m j} \psi_{l}=0$ for $l^{k} \neq m$.

Remark 4.3. It is not difficult to show that $f \in l^{2}(\mathbb{N}) \mapsto W_{Y}(f) \in \mathscr{U}\left(H_{Y}\right)$ is even strongly continuous (see [10] for a proof).

\section{\$5. Insertion about Decompositions with the Same Diagonal Algebra}

Let $\mathscr{A}$ be a unital $C^{*}$-algebra, $\omega$ a state of $\mathscr{A}$, and $\mathscr{C}$ an abelian von Neumann subalgebra of $\pi_{\omega}(\mathscr{A})^{\prime}$. Moreover, let $v$ be the orthogonal measure in $\mathscr{S}(\mathscr{A})$ associated with $\omega$ and $\mathscr{C}$.

5. 1 Theorem. Let

$$
\pi_{\omega}=\int_{Z}^{\oplus} \pi(\zeta) d \mu(\zeta)
$$

be a (with respect to $\xi_{\omega}$ ) normalized decomposition of $\pi_{\omega}$ into representations $\pi(\zeta): \mathscr{A} \rightarrow \mathscr{L}(H(\zeta))$ of $\mathscr{A}$ such that $\mathscr{C}$ is transformed into the diagonal algebra of the direct integral $\int_{Z}^{\oplus} H(\zeta) d \mu(\zeta)$ (in the sense of Definition 2.1). Let $\xi_{\omega}$ $=\int_{Z}^{\oplus} \xi(\zeta) d \mu(\zeta)$ be the corresponding decomposition of $\xi_{\omega}$, for $\zeta \in \mathbb{Z}$ let $\varphi(\zeta)$ be the state of $\mathscr{A}$ belonging to $\pi(\zeta)$ and $\xi(\zeta)$. Moreover, let $\Sigma$ be the $\sigma$-field, on which 
the measure $\mu$ is defined, let $v_{0}$ be the restriction of $v$ to a Baire measure on $\mathscr{S}(\mathscr{A})$, and $\mathscr{B}_{0}$ the $\sigma$-field of Baire sets on $\mathscr{S}(\mathscr{A})$.

Then $T: Z \rightarrow \mathscr{S}(\mathscr{A}), \zeta \mapsto \varphi(\zeta)$, is $\Sigma-\mathscr{B}_{0}$-measurable, and $v_{0}$ is the image of $\mu$ under $T$.

Proof. In several steps:

(a) Let $U$ be the Hilbert space isomorphism which is composed of both the canonical isomorphisms, transforming $\int_{\mathscr{S}(\mathscr{A})}^{\oplus} H_{\varphi} d v(\varphi)$ into $H_{\omega}$ and $H_{\omega}$ into $\int_{Z}^{\oplus} H(\zeta) d \mu(\zeta)$, resp.. For $a \in \mathscr{A}$,

$$
U \int_{\mathscr{S}(\mathscr{A})}^{\oplus} \varphi(a) \mathbb{1} d v(\varphi) U^{*}=\int_{Z}^{\oplus} \varphi(\zeta)(a) \mathbb{1} d \mu(\zeta)
$$

holds.

For: Let $P$ be the orthogonal projection onto $\left[\mathscr{C}_{\omega}\right]$ in $H_{\omega}$. For $C \in \mathscr{C}^{\prime}$ there is a unique operator $\Phi(C) \in \mathscr{C}$ such that $P C P=\Phi(C) P$ (cf. [6], §2.). In order to get the assertion (10) we will show that for $a \in \mathscr{A}$

$$
\Phi\left(\pi_{\omega}(a)\right)=\int_{\mathscr{S}(\mathscr{A})}^{\oplus} \varphi(a) \mathbb{1} d v(\varphi)
$$

if we identify $H_{\omega}$ and $\int_{\mathscr{S}(\mathscr{A})}^{\oplus} H_{\varphi} d v(\varphi)$, and that

$$
\Phi\left(\pi_{\omega}(a)\right)=\int_{Z}^{\oplus} \varphi(\zeta)(a) \mathbb{1} d v(\zeta)
$$

if we identify $H_{\omega}$ and $\int_{Z}^{\oplus} H(\zeta) d \mu(\zeta)$. It suffices to establish (12); (11) follows in the same way. So we have to prove

$$
P \pi_{\omega}(a) \eta=\int_{Z}^{\oplus} \varphi(\zeta)(a) \mathbb{1} d \mu(\zeta) \cdot \eta \quad \text { for every } \eta \in \mathscr{C} \xi_{\omega}
$$

We can easily check this relation after having shown that

$$
P=\int_{z}^{\oplus} P(\zeta) d \mu(\zeta)
$$

where $P(\zeta)$ is the projection onto $\mathbb{C} \xi(\zeta)$ for $\zeta \in Z$.

$$
\text { Obviously, } \int_{Z}^{\oplus} P(\zeta) d \mu(\zeta) \text { is well defined, }\left(\int_{Z}^{\oplus} P(\zeta) d \mu(\zeta)\right)\left(H_{\omega}\right) \subseteq\left[\mathscr{C} \xi_{\omega}\right] \text {, and }
$$


$\int_{Z}^{\oplus} P(\zeta) d \mu(\zeta) \cdot \eta=\eta$ for $\eta \in \mathscr{C} \xi_{\omega}$. It remains to prove that $\int_{Z}^{\oplus} P(\zeta) d \mu(\zeta) \cdot \eta \in\left(\mathscr{C} \xi_{\omega}\right)^{\perp}$ holds for $\eta=\int_{Z}^{\oplus} \eta(\zeta) d \mu(\zeta) \in\left(\mathscr{C} \xi_{\omega}\right)^{\perp}$. We get this from

$$
\begin{aligned}
& \left\langle\int_{Z}^{\oplus} P(\zeta) d \mu(\zeta) \cdot \eta, \int_{Z}^{\oplus} h(\zeta) \xi(\zeta) d \mu(\zeta)\right\rangle= \\
& \quad=\int_{Z}\langle\langle\eta(\zeta), \xi(\zeta)\rangle \xi(\zeta), h(\zeta) \xi(\zeta)\rangle d \mu(\zeta)= \\
& \quad=\left\langle\int_{Z}^{\oplus} \eta(\zeta) d \mu(\zeta), \int_{Z}^{\oplus} h(\zeta) \xi(\zeta) d \mu(\zeta)\right\rangle=0
\end{aligned}
$$

for $h \in L^{\infty}(Z, \mu)$.

(b) For $a \in \mathscr{A}_{+}$and an interval $I$ in $\mathbb{R}_{0}^{+}$we define $B_{a, I}:=\{\varphi \in \mathscr{S}(\mathscr{A}): \varphi(a) \in \mathbb{I}\}$ and $C_{a, I}:=T^{-1}\left(B_{a, I}\right)=\{\zeta \in Z: \varphi(\zeta)(a) \in I\}$. For $I=\left[0, s\left[(s>0), C_{a, I}\right.\right.$ is $\mu-$ measurable, and

$$
U \int_{\mathscr{S}(\mathscr{A})}^{\oplus} \chi_{B_{a, I}}(\varphi) \mathbb{1} d \nu(\varphi) U^{*}=\int_{Z}^{\oplus} \chi_{C_{a, I}}(\zeta) \mathbb{1} d \mu(\zeta)
$$

For: Since $\int_{\mathscr{S}(\mathscr{A})}^{\oplus} \chi_{B_{a, I}}(\varphi) \mathbb{1} d v(\varphi)$ is a projection, there is a set $D_{a, I} \in \Sigma$ satisfying (13) where $C_{a, I}$ is replaced by $D_{a, I}$. From (a) we get

$$
U \int_{\mathscr{S}(\mathscr{A})}^{\oplus} \chi_{B_{a, I}}(\varphi)(s-\varphi(a)) \mathbb{1} d v(\varphi) U^{*}=\int_{Z}^{\oplus} \chi_{D_{a, I}}(\zeta)(s-\varphi(\zeta)(a)) \mathbb{1} d \mu(\zeta) .
$$

The operator on the left is positive, therefore the operator on the right is positive, too. We obtain

$$
\varphi(\zeta)(a) \leq s \text { for almost every } \zeta \in D_{a, I} .
$$

Since the operator

$$
\int_{\mathscr{S}(\mathscr{A})}^{\oplus}\left(1-\chi_{B_{a, I}}(\varphi)\right)(\varphi(a)-s) \mathbb{1} d v(\varphi)
$$

is positive, we conclude as before that $\varphi(\zeta)(a) \geq s$ for almost every $\zeta \in \mathbb{Z} \backslash D_{a, I}$. After a modification on a negligible set we get

$$
C_{a,[0, s[} \subseteq D_{a, I} \subseteq C_{a,[0, s]} .
$$

For $n \in \mathbb{N}$ let $I_{n}:=[0, s(1-1 / n)[$. We have

$$
U \int_{\mathscr{S}(\mathscr{A})}^{\oplus} \chi_{B_{a, I}}(\varphi) \mathbb{H} d v(\varphi) U^{*}=U\left(\sup _{n \in \mathbb{N}} \int_{\mathscr{S}(\mathscr{A})}^{\oplus} \chi_{B_{a, I_{n}}}(\varphi) \mathbb{1} d v(\varphi)\right) U^{*}=
$$




$$
=\sup _{n \in \mathbb{N}} \int_{Z}^{\oplus} \chi_{D_{a, I_{n}}}(\zeta) \mathbb{1} d \mu(\zeta) .
$$

From (14) (applied to $I_{n}$ ), $C_{a, I}=\bigcup_{n=1}^{\infty} D_{a, I_{n}}$ follows. Thus $C_{a, I}$ is $\mu$-measurable, and

$$
\sup _{n \in \mathbb{N}} \int_{Z}^{\oplus} \chi_{D_{a, I_{n}}}(\zeta) \mathbb{1} d \mu(\zeta)=\int_{Z}^{\oplus} \chi_{C_{a, I}}(\zeta) \mathbb{1} d \mu(\zeta) .
$$

From this and (15) the assertion follows.

(c) So

$$
U \int_{\mathscr{S}(\mathscr{A})}^{\oplus} \chi_{E}(\varphi) \mathbb{1} d v(\varphi) U^{*}=\int_{Z}^{\oplus} \chi_{T^{-1}(E)}(\zeta) \mathbb{R} d \mu(\zeta)
$$

holds for every $E=B_{a,[0, s[}$. Similar arguments establish (16) for $E=B_{a,[0, s]}$ $(s>0)$. Now it is easy to see that (16) is satisfied for every

$$
\begin{aligned}
E \in \mathscr{E}:=\left\{\bigcap_{n=1}^{m} B_{a_{n}, I_{n}}: m \in \mathbb{N}, a_{n} \in \mathscr{A}_{+}, I_{n}\right. & \text { a relatively open interval } \\
& \text { in } \left.\mathbb{R}_{0}^{+}, n=1, \ldots, m .\right\} .
\end{aligned}
$$

Thus for $E \in \mathscr{E}$ we obtain

$$
\begin{aligned}
v(E) & =\left\langle\int_{\mathscr{S}(\mathscr{A})}^{\oplus} \chi_{E}(\varphi) \mathbb{1} d v(\varphi) \int_{\mathscr{S}(\mathscr{A})}^{\oplus} \xi_{\varphi} d v(\varphi), \int_{\mathscr{S}(\mathscr{A})}^{\oplus} \xi_{\varphi} d \nu(\varphi)\right\rangle \\
& =\left\langle\int_{\mathscr{S}(\mathscr{A})}^{\oplus} \chi_{E}(\varphi) \mathbb{1} d v(\varphi) U^{*} \int_{Z}^{\oplus} \xi(\zeta) d \mu(\zeta), U^{*} \int_{Z}^{\oplus} \xi(\zeta) d \mu(\zeta)\right\rangle \\
& =\left\langle\int_{Z}^{\oplus} \chi_{T^{-1}(E)}(\zeta) \mathbb{1} d \mu(\zeta) \int_{Z}^{\oplus} \xi(\zeta) d \mu(\zeta), \int_{Z}^{\oplus} \xi(\zeta) d \mu(\zeta)\right\rangle=\mu\left(T^{-1}(E)\right)
\end{aligned}
$$

Since the $\sigma$-field $\mathscr{B}_{0}$ is generated by $\mathscr{E}$, and $\mathscr{E}$ is closed with respect to finite intersections, $v(B)=\mu\left(T^{-1}(B)\right)$ holds for every $B \in \mathscr{B}_{0}$.

We mainly need the following corollary.

Corollary 5.2. Additionally, let us suppose that $\mathscr{A}$ is a separable $C^{*}$ algebra. Let

$$
\pi_{\omega}=\int_{Z}^{\oplus} \pi(\zeta) d \mu(\zeta) \text { and } \pi_{\omega}=\int_{X}^{\oplus} \rho(\beta) d \lambda(\beta)
$$

be two decompositions of $\pi_{\omega}$ with diagonal algebra $\mathscr{C}$, and $H_{\omega}=\int_{Z}^{\oplus} H(\zeta) d \mu(\zeta)$ and $H_{\omega}=\int_{X}^{\oplus} K(\beta) d \lambda(\beta)$, resp., the corresponding decompositions of $H_{\omega}$. Moreover, 
let the first decomposition of $\pi_{\omega}$ be normalized with respect to $\xi_{\omega}$; let $\int_{X}^{\oplus} K(\beta) d \lambda(\beta)$ be a direct integral in the sense of $J$. Dixmier, let $X$ be a standard Borel space and $\lambda$ a $\sigma$-finite measure on $X$.

Then for almost every $\zeta \in Z$ there is a $\beta \in X$ such that $\pi(\zeta)$ and $\rho(\beta)$ are unitarily equivalent.

Remarks. (i) From the assumptions above it follows that $\int_{Z}^{\oplus} H(\zeta) d \mu(\zeta)$ is a direct integral in the sense of $J$. Dixmier.

(ii) If $Z$ is a standard Borel space, too, the corollary is an immediate conclusion from [2], Proposition 8.2.4, but we are interested in the general case.

Proof. Let $v$ be the orthogonal measure in $\mathscr{S}(\mathscr{A})$ associated with $\omega$ and $\mathscr{C}$, and let $\pi_{\omega}=\int_{\mathscr{S}(\mathscr{A})}^{\oplus} \pi_{\varphi} d v(\varphi)$ the corresponding decomposition of $\pi_{\omega}$. Since $\mathscr{S}(\mathscr{A})$ is compact and metrizable, $\mathscr{S}(\mathscr{A})$ is a standard Borel space, and the direct integral can be interpreted in the sense of J. Dixmier. Let us compare this decomposition of $\pi_{\omega}$ with $\pi_{\omega}=\int_{X}^{\oplus} \rho(\beta) d \lambda(\beta)$. The Proposition 8.2.4 in [2] mentioned above implies that there are a $v$-negligible set $N$, a $\lambda$-negligible set $N_{1}$, and a Borel isomorphism $S: \mathscr{S}(\mathscr{A}) \backslash N \rightarrow X \backslash N_{1}$ such that $\pi_{\varphi}$ is unitarily equivalent to $\rho(S(\varphi))$ for $\varphi \in \mathscr{S}(\mathscr{A}) \backslash N$. Since the set of Baire sets in $\mathscr{S}(\mathscr{A})$ is equal to the set of Borel sets, from Theorem 5.1 we get that $T^{-1}(\mathscr{S}(\mathscr{A}) \backslash N)$ is $\mu$ measurable, $\mu\left(T^{-1}(\mathscr{S}(\mathscr{A}) \backslash N)\right)=1$, and that for $\zeta \in T^{-1}(\mathscr{S}(\mathscr{A}) \backslash N) \pi(\zeta)$ is unitarily equivalent to $\pi_{\varphi(\zeta)}$ and therefore to $\rho(S(\varphi(\zeta)))$.

\section{\$6. The Example, Part 2}

Proposition 6.1. There is no decomposition

$$
W_{Y}=\int_{Z}^{\Gamma} W(\zeta) d \mu(\zeta)
$$

of $W_{Y}$ into regular representations $W(\zeta)$ of the $C C R$ which are defined over $l^{2}(\mathbb{N})$ such that the diagonal algebra of the direct integral is maximal abelian in $\left\{W_{Y}(f): f \in l^{2}(\mathbb{N})\right\}^{\prime}$.

Proof. In several steps:

(a) Let us suppose that

$$
W_{Y}=\int_{Z}^{\Gamma} W(\zeta) d \mu(\zeta)
$$


is a maximal abelian decomposition of $W_{Y}$ into regular representations $W(\zeta): l^{2}(\mathbb{N}) \rightarrow \mathscr{U}(H(\zeta))$

Let $\pi_{Y}\left(\pi(\zeta)\right.$, resp.) be the representation of $\mathscr{A}\left(l^{2}(\mathbb{N})\right)$ associated with $W_{Y}$ $\left(W(\zeta)\right.$, resp.). Let $\mathscr{A}\left(V_{0}, \mathbb{Q}\right)$ be the $C^{*}$-subalgebra of $\mathscr{A}\left(l^{2}(\mathbf{N})\right)$ generated by

$$
\left\{w\left(\sum_{n=1}^{m} \lambda_{n} e_{n}\right): m \in \mathbb{N}, \lambda_{n} \in \mathbf{Q}+i \mathbf{Q}, n=1, \ldots, m\right\}
$$

obviously $\mathscr{A}\left(V_{0}, \mathbb{Q}\right)$ is separable. Let $\pi_{Y, \mathbb{Q}}\left(\pi(\zeta)_{\mathbf{Q}}\right.$, resp.) be the restriction of $\pi_{Y}$ $\left(\pi(\zeta)\right.$, resp.) on $\mathscr{A}\left(V_{0}, \mathbb{Q}\right)$. We obtain the decomposition

$$
\pi_{Y, \mathbf{Q}}=\int_{Z}^{\Gamma} \pi(\zeta)_{\mathbf{Q}} d \mu(\zeta)
$$

of $\pi_{Y, \mathbb{Q}}$. Since

$$
\pi_{Y, \mathbb{Q}}\left(\mathscr{A}\left(V_{0}, \mathbb{Q}\right)\right)^{\prime \prime}=\left\{W_{Y}^{0}(f): f \in V_{0}\right\}^{\prime \prime}=\left\{W_{Y}(f): f \in l^{2}(\mathbb{N})\right\}^{\prime \prime},
$$

$\xi_{Y}$ is a cyclic vector for $\pi_{Y, \mathbb{Q}}$, and the diagonal algebra in $\int_{Z}^{\Gamma} H(\zeta) d \mu(\zeta)$ is $\pi_{Y, \mathbb{Q}}\left(\mathscr{A}\left(V_{0}, \mathbb{Q}\right)\right)^{\prime}$ (see Proposition $4.2(\mathrm{ii})$ ).

(b) Our next intension is to normalize the decomposition (17).

Let $\xi_{Y}=\int_{Z}^{\Gamma} \xi(\zeta) d \mu(\zeta)$ be the corresponding decomposition of $\xi_{Y}$. For $\zeta \in Z$ let $K(\zeta):=\left[\pi(\zeta)_{\mathbb{Q}}\left(\mathscr{A}\left(V_{0}, \mathbb{Q}\right)\right) \xi(\zeta)\right]$. Since $\xi_{Y}$ is cyclic for $\pi_{Y, \mathbb{Q}}$, almost every $\eta(\zeta)$ belongs to $K(\zeta)$ for every $\eta=(\eta(\zeta))_{\zeta \in Z} \in \Gamma$. Thus $\int_{Z}^{\Gamma} H(\zeta) d \mu(\zeta)$ is isomorphic to $\int_{Z}^{\tilde{\Gamma}} K(\zeta) d \mu(\zeta)$ in a canonical way, where $\tilde{\Gamma}=\Gamma \cap \prod_{\zeta \in Z} K(\zeta)$ is the set of square integrable vector fields in $\int_{Z}^{\tilde{\Gamma}} K(\zeta) d \mu(\zeta)$.

We can assume that all the $K(\zeta)$ 's are not equal to zero. (Otherwise we remove every $\zeta \in Z$ satisfying $K(\zeta)=0$ from $Z$.) Instead of $\mu$ we can use a suitable measure $\tilde{\mu}$ equivalent to $\mu$, and transform $\int_{Z}^{\tilde{\Gamma}} K(\zeta) d \mu(\zeta)$ into $\int_{Z}^{\Delta} K(\zeta) d \tilde{\mu}(\zeta)$ such that in the corresponding decomposition

$$
\xi_{Y}=\int_{Z}^{\Delta} \tilde{\xi}(\zeta) d \tilde{\mu}(\zeta)
$$

of $\xi_{Y}$ every $\tilde{\zeta}(\zeta)$ is equal to $\frac{\xi(\zeta)}{\|\xi(\zeta)\|}$. (For details see [10].)

In this way we obtain the decomposition 


$$
\pi_{Y, \mathbb{Q}}=\int_{Z}^{\Delta} \tilde{\pi}(\zeta)_{\mathbb{Q}} d \mu(\zeta)
$$

of $\pi_{Y, \mathbb{Q}}$, where $\tilde{\pi}(\zeta)_{\mathbb{Q}}$ denotes the restriction of $\pi(\zeta)_{\mathbb{Q}}$ on $K(\zeta)$. It is easy to show that the diagonal algebra is $\pi_{Y, \mathbb{Q}}\left(\mathscr{A}\left(V_{0}, \mathbb{Q}\right)\right)^{\prime}$.

(c) For $\alpha \in Y$ let $\pi_{\alpha}$ be the representation of $\mathscr{A}\left(V_{\alpha}\right)$ corresponding to $W_{\alpha}$ and $\pi_{\alpha, \mathbb{Q}}$ the restriction of $\pi_{\alpha}$ on $\mathscr{A}\left(V_{0}, \mathbb{Q}\right)$. It is easy to recognize that

$$
\pi_{Y, \mathbb{Q}}=\int_{Y}^{\oplus} \pi_{\alpha, \mathbb{Q}} d \alpha
$$

is a decomposition of $\pi_{Y, \mathbb{Q}}$ such that $\pi_{Y, \mathbb{Q}}\left(\mathscr{A}\left(V_{0}, \mathbb{Q}\right)\right)^{\prime}$ is the diagonal algebra in the direct integral. Let us compare the decompositions (18) and (19): Corollary 5.2 implies that almost every $\tilde{\pi}(\zeta)_{\mathbb{Q}}$ is unitarily equivalent to a representation $\pi_{\alpha, \mathbb{Q}}$. This is a contradiction, since $\pi_{\alpha, \mathbb{Q}}$ cannot be extended to a regular representation of $\mathscr{A}\left(l^{2}(\mathbb{N})\right)$.

Proposition 6.1 suggests that $W_{Y}$ cannot be decomposed into irreducible regular representations of the $\mathrm{CCR}$ over $l^{2}(\mathbb{N})$. For example, our result shows that it is not possible to extend the representations of the fibers appearing in the decomposition of Theorem 2.3 to irreducible regular representations over $l^{2}(\mathbb{N})$, and so to get a decomposition of $W_{Y}$ into irreducible regular representations of the $\mathbb{C} \mathbb{R}$ over $l^{2}(\mathbb{N})$.

But there remains the question whether there is a decomposition of $W_{Y}$ into irreducible regular representations of the $C C R$ over $l^{2}(\mathbb{N})$ such that the diagonal algebra is not maximal abelian in $W_{Y}\left(l^{2}(\mathbb{N})\right)^{\prime}$.

We cannot exclude this possibility completely for the following reason: In contrast to the case of separable $C^{*}$-algebras there is a representation $\pi$ of a nonseparable $C^{*}$-algebra $\mathscr{A}$ possessing a decomposition into irreducible fibers such that the diagonal algebra is not maximal abelian in $\pi(\mathscr{A})^{\prime}$. A still unpublished example of such a kind was constructed by R.W. Henrichs. Is it possible that this phenomen also appears in the case of the representation $\pi_{Y}$ of $\mathscr{A}\left(l^{2}(\mathbb{N})\right)$ ?

Remark 6.2. It can be shown (see [10], Proposition 3.4.2) that there is no decomposition fo $W_{Y}$ into factorial representations $W(\zeta)$ of the $\operatorname{CCR}$ over $l^{2}(\mathbb{N})$, for which

$$
f \in l^{2}(\mathbb{N}) \longrightarrow W(\zeta)(f)
$$

is strongly continuous. So it is impossible to transfer the strong continuity of $W_{Y}$ (see Remark 4.3) to the representations of the fibers. 


\section{Ackmowledgememts}

It is a pleasure to thank Prof. R.W. Henrichs for suggesting the problem and encouraging the progress of this work. I am also grateful to $\mathbf{M}$. Binder for his technical advice.

\section{Referemces}

[1] Dixmier, J., Von Neumann algebras, North-Holland, Amsterdam-New York-Oxford, 1981.

[2] - , C $C^{*}$-algebras, North-Holland, Amsterdam-New York-Oxford, 1977.

[3] Guichardet, A., Tensor product of $C^{*}$-algebras, Part II, Infinite tensor products, Aarhus, Lecture Note Series, 13 (1969).

[4] Hegerfeldt, G.C., Decomposition into irreducible representations for the canonical commutation relations, Nuovo Cimento B, 4 (1971), 225-244.

[5] - Representations of the canonical commutation relations, in Functional analysis: Survey's and results II (Bierstedt, K.-D., Fuchssteiner, B., eds.), North-Holland (1980), 149-164.

[6] Henrichs, R.W., Decomposition of invariant states and nonseparable $C^{*}$-algebras, Publ. Res. Inst. Math. Sci., 18 (1982), 159-181.

[7] Klauder, J.R., McKenna, J., Woods E.J., Direct-product representations of the canonical commutation relations, J. Math. Phys., 7 (1966), 822-828.

[8] von Neumann, J., On infinite direct products, Compositio Math., 6 (1938), 1-77.

[9] Schaflitzel, R., Some particle representations of the canonical commutation relations, Rep. Math. Phy's., 25 (1988), 329-344.

[10] - Zerlegungen regulärer Darstellungen der kanonischen Vertauschungsrelationen, Dissertation, Technische Universität München, 1988.

[11] Streit, L., Test function spaces for direct-product representations of the canonical commutation relations, Comm. Math. Phys., 4 (1967), 22-31.

[12] Wils, W., Direct integrals of Hilbert spaces I, Math. Scand., 26 (1970), 73-88.

[13] Woods, E.J., Continuity properties of the representations of the canonical commutation relations, Comm. Math. Phys., 17 (1970), 1-20. 
